\title{
Very Large Scale Optical Interconnect Systems For Different Types of Optical Interconnection Networks
}

\author{
Dr. Ahmed Nabih Zaki Rashed \\ Electronics and Electrical Communications Engineering Department \\ Faculty of Electronic Engineering, Menouf 32951, Menoufia University, EGYPT \\ E-mail: ahmed 733@yahoo.com
}

\begin{abstract}
The need for scalable systems in market demands in terms of lower computing costs and protection of customer investment in computing: scaling up the system to quickly meet business growth is obviously a better way of protecting investment: hardware, software, and human resources. A scalable system should be incrementally expanded, delivering linear incremental performance with a near linear cost increase, and with minimal system redesign (size scalability), additionally, it should be able to use successive, faster processors with minimal additional costs and redesign (generation scalability). On the architecture side, the key design element is the interconnection network. The interconnection network must be able to increase in size using few building blocks and with minimum redesign, deliver a bandwidth that grows linearly with the increase in system size, maintain a low or (constant) latency, incur linear cost increase, and readily support the use of new faster processors. The major problem is the ever-increasing speed of the processors themselves and the growing performance gap between processor technology and interconnect technology. Increased central processing unit (CPU) speeds and effectiveness of memory latency-tolerating techniques.
\end{abstract}

Index Terms-Bisection width, Node degree, Performance evolution, Optical interconnection network, Network parameters, Scalability.

\section{INTRODUCTION}

Numerous applications for interconnection networks with extremely high throughput and low latency have been studied previously, including high-performance computing and telecommunications core routing [1]. Optical interconnection networks (OINs) are able to meet many of these system requirements by leveraging the large bandwidth afforded by fiber-optic and photonic technologies. Multiple stage (or multistage) interconnection networks (MINs) offer topological advantages such as scalability and modularity owing to their composition from hundreds or thousands of similar switching node building blocks [2]. In OIN systems, most of the complexity and cost is attributable to the photonic switching elements. When multiple wavelength data is employed, these switching elements must execute space switching in addition to wavelength switching. Many switching node designs based on this technique have been proposed and most are implemented with semiconductor optical amplifier (SOA) switching gates. The effectiveness of parallel computers is often determined by its communication network. The interconnection network is an important component of a parallel processing system. A good interconnection network should have less topological network cost and keep the network diameter as shorter as possible [3].

In the present study, an important issues in the design of optical interconnection networks for high performance computing and communication systems is scalability. As well as different types of optical interconnection networks for scalable high performance communication and computing systems have been modeled, investigated and a analyzed numerically and parametrically over wide range of the affecting parameters. Optical interconnection networks is a promising design and performance parameters alternative for future systems. Numerous configurations with different degrees of optics, optoelectronics, network diameter, node degree, bisection width, scalability, transmission capacity, cost evolution, and electronics have been proposed.

\section{CURRENT ARChiteCtURES FOR SCALABLE PARALLEL COMPUTING SYSTEMS}

\section{1. SCALABLE OPTICAL CROSSBAR CONNECTED INTERCONNECTION NETWORK (SCON)}

SOCN stands for "scalable optical crossbar connected interconnection Network". A two-level hierarchical network. The lowest level consists of clusters of $\mathrm{n}$ processors connected via local WDM intra-cluster all-optical crossbar subnetwork. Multiple (c) clusters are connected via similar WDM intra-cluster all-optical crossbar that connects all processors in a single cluster to all processors in a remote cluster. The inter-cluster crossbar connections can be rearranged to form various network topologies [5]. 


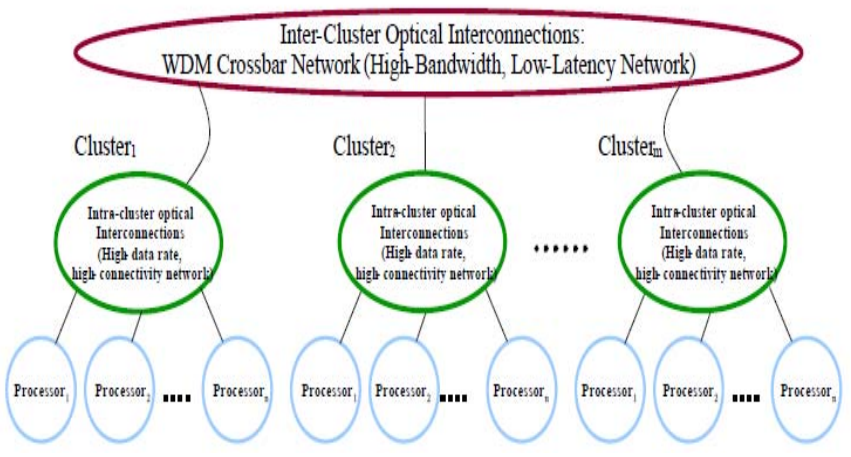

Fig. 1. Scalable optical crossbar connected interconnection network [6].

Both the intra-cluster and inter-cluster subnetworks are WDM-based optical crossbar interconnects. Architecture based on wavelength reuse. The SOCN class of networks are based on WDM all-optical crossbar networks. The benefits of crossbar networks are fully connected, minimum potential latency, highest potential bisection bandwidth, can be used as a basis for multistage and hierarchical networks. But disadvantages of crossbar networks are $\mathrm{O}\left(\mathrm{N}^{2}\right)$ complexity, difficult to implement in electronics, $\mathrm{N}^{2}$ wires and switches required, rise time and timing skew become a limitation for large crossbar interconnects, optics and WDM can be used to implement a crossbar with $\mathrm{O}(\mathrm{N})$ complexity [6].

\section{1. 1. OPTICAL CROSSBAR CONNECTED CLUSTER NETWORK (OC3N)}

Every cluster is connected to every other cluster via a single send/receive optical fiber pair. Each optical fiber pair supports a wavelength division multiplexed fullyconnected crossbar interconnect. Full connectivity is provided: every processor in the system is directly connected to every other processor with a relatively simple design. Inter-cluster bandwidth and latencies similar to intra-cluster bandwidth and latencies. Far fewer connections are required compared to a traditional crossbar [7].

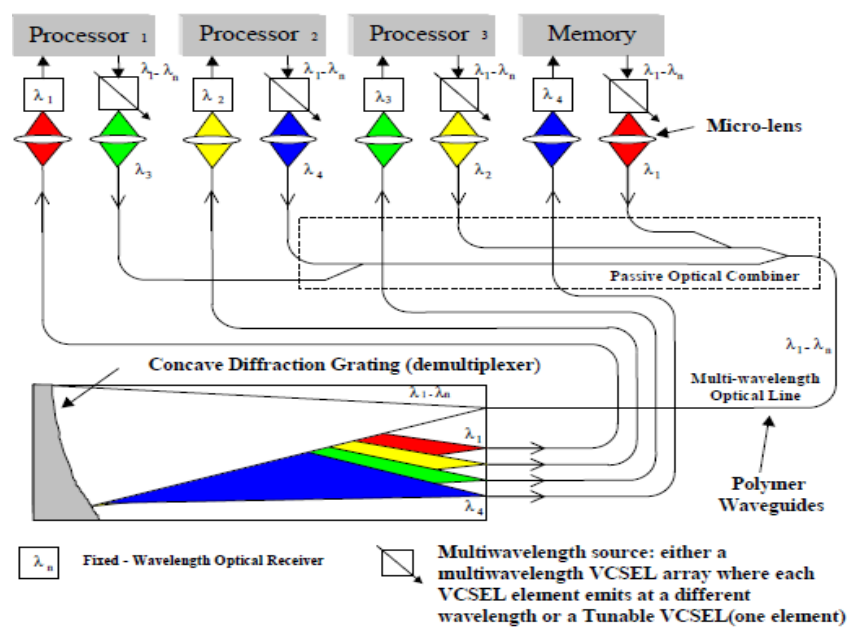

Fig. 2. WDM optical crossbar implementation [7].

Each processor contains a single integrated tunable vertical cavity surface emitting laser (VCSEL) or a VCSEL array, and one optical receiver. Each VCSEL is coupled into a personal board integrated polymer waveguide. The waveguides from all processors in a cluster are routed to a polymer waveguide based optical binary tree combiner. The combined optical signal is routed to a free space diffraction grating based optical demultiplexer. The demultiplexed optical signals are routed back to the appropriate processors [7]. The $\mathrm{OC}^{3} \mathrm{~N}$ topology efficiently utilizes wavelength division multiplexing throughout the network, so it could be used to construct relatively large (hundreds of processors) fully connected networks with a reasonable cost. One of the advantages of a hierarchical network architecture is that the various topological layers typically can be interchanged without effecting the other layers. The lowest level of the SOCN is a fully connected crossbar. The second (and highest) level can be interchanged with various alternative topologies as long as the degree of the topology is less than or equal to the cluster node degree are crossbar, hypercube, tree, and ring [8].

\section{1. 2. OPTICAL HYPERCUBE CONNECTED CLUSTER NETWORK (OHC2N)}

Processors within a cluster are connected via a local intra-cluster WDM optical crossbar. Clusters are connected via inter-cluster WDM optical links. Each processor in a cluster has full connectivity to all processors in directly connected clusters. The intercluster crossbar connecting clusters are arranged in a hypercube configuration [9].

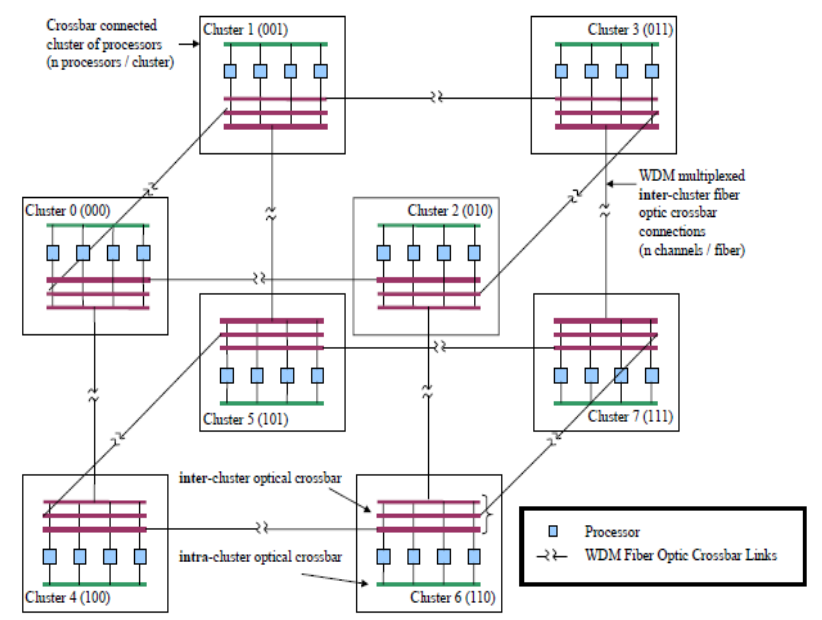

Fig. 3. Optical hypercube connected cluster network [9].

The $\mathrm{OHC}^{2} \mathrm{~N}$ does not impose a fully connected topology, but efficient use of WDM allows construction of very large-scale (thousands of processors) networks at a reasonable cost [9].

\section{THEORETICAL MODEL ANALYSIS \\ III. 1. PERFORMANCE PARAMETERS OF OC3N AND OHC2N NETWORKS}

\section{1. 1. DIAMETER AND LINK COMPLEXITY}

The diameter of the network is defined as the maximum distance between two any processors in the 
network. Also defined as For each possible pair of nodes in the network there exist a shortest path. The diameter is defined as the number of hops over the longest of these shortest paths [7]. The number of nodes for different networks $\left(\mathrm{OC}^{3} \mathrm{~N}\right.$ and $\left.\mathrm{OHC}^{2} \mathrm{~N}\right)$ are:

$$
\begin{aligned}
& N_{C}=n \times c, \text { for }\left(\mathrm{OC}^{3} \mathrm{~N} \text { network }\right) \\
& N_{H}=n \times 2^{d}, \text { for }\left(\mathrm{OHC}^{2} \mathrm{~N} \text { network }\right)
\end{aligned}
$$

Where $\mathrm{n}$ is the number of processors per cluster, $\mathrm{c}$ is the number of clusters, $d$ is the network diameter and is:

$$
\begin{aligned}
& d=\text { unity }, \quad \text { for }\left(\mathrm{OC}^{3} \mathrm{~N} \text { network }\right) \\
& d=\log _{2}\left(\frac{N}{n}\right), \text { for }\left(\mathrm{OHC}^{2} \mathrm{~N} \text { network }\right)
\end{aligned}
$$

Where the link complexity or node degree is defined as the number of physical links per node. Also can be defined as the number of links that connects a node to its nearest neighbors. The node degree can either be constant for the whole network or differ between the nodes [8]. Where the node degree $\mathrm{K}$ for different networks $\left(\mathrm{OC}^{3} \mathrm{~N}\right.$ and $\mathrm{OHC}^{2} \mathrm{~N}$ ) are:

$$
\begin{aligned}
& K=c=\frac{N}{n}, \text { for }\left(\mathrm{OC}^{3} \mathrm{~N} \text { network }\right) \\
& K=d+1, \text { for }\left(\mathrm{OHC}^{2} \mathrm{~N} \text { network }\right)
\end{aligned}
$$

\section{1. 2. BISECTION BANDWIDTH}

The bisection of a system is the section that divides the system into two halves with equal number of nodes. The bisection bandwidth is the aggregated bandwidth over the links that cross the bisection. In asymmetric systems the number of links across the bisection depends on where the bisection is drawn. However, since the bisection bandwidth is a worst case metric, the bisection leading to the smallest bisection bandwidth should be chosen [9]. Where the number of links L for different networks $\left(\mathrm{OC}^{3} \mathrm{~N}\right.$ and $\left.\mathrm{OHC}^{2} \mathrm{~N}\right)$ are given by:

$$
\begin{aligned}
& L_{C}=0.5\left(\frac{N^{2}}{n^{2}}-\frac{N}{n}\right), \text { for (OC }{ }^{3} \mathrm{~N} \text { network) } \\
& L_{H}=\frac{1}{2} 2^{d} d=\frac{N}{2 n} \log _{2} \frac{N}{n} \quad, \text { for }\left(\mathrm{OHC}^{2} \mathrm{~N} \text { network }\right)
\end{aligned}
$$

In the same way, the bisection bandwidth for different networks $\left(\mathrm{OC}^{3} \mathrm{~N}\right.$ and $\left.\mathrm{OHC}^{2} \mathrm{~N}\right)$ are given by [11]:

$$
\begin{aligned}
& B . W_{C}=\frac{N^{2}}{4}=\frac{(n \times c)^{2}}{4}, \text { for }\left(\mathrm{OC}^{3} \mathrm{~N} \text { network }\right) \\
& \text { B. } W_{H}=n 2^{d-1}=\frac{N}{2}, \text { for }\left(\mathrm{OHC}^{2} \mathrm{~N} \text { network }\right)
\end{aligned}
$$

\section{1. 3. AVERAGE MESSAGE DISTANCE}

The average message distance in the network is defined as the average number of the links that a message should travel between any nodes. Let $\mathrm{N}_{\mathrm{i}}$ is the number of nodes at distance $i$, then the average distance $\bar{l}$ is defined as [12]:

$$
\bar{l}=\frac{1}{N-1} \sum_{i=1}^{n} i N_{i},
$$

Therefore the average message distance for different networks $\left(\mathrm{OC}^{3} \mathrm{~N}\right.$ and $\left.\mathrm{OHC}^{2} \mathrm{~N}\right)$ are given by $[11,12]$ :

$$
\bar{l}_{C}=\text { unity, for }\left(\mathrm{OC}^{3} \mathrm{~N} \text { network }\right)
$$

$$
\bar{l}_{H}=\frac{1}{N-1}\left[0.5 N \log _{2}\left(\frac{N}{n}\right)+(n-1)\right],\left(\mathrm{OHC}^{2}\right)
$$

\section{1. 4. COST SCALABILITY OF DIFFERENT NETWORKS}

A major advantage of a SOCN architecture is the reduced hardware part count compared to more traditional network topologies. The cost scalability of the two networks can be estimated based on the number of the tunable VCSELs V, number of detectors per processor $\mathrm{D}$, and waveguides per processor $\mathrm{W}$, and number of demultiplexers in the cluster $\mathrm{M}$ as:

Cost $_{C}=n V+n D+n W+c M, \$$ for (OC ${ }^{3} \mathrm{~N}$ network) (14)

$\operatorname{Cost}_{H}=\log _{2}(n V+n D+n W+c M), \$$ for $\left(\mathrm{OHC}^{2} \mathrm{~N}\right.$ network $)$

\section{1. 5. TRANSMISSION CAPACITY OF DIFFERENT NETWORKS}

The transmission capacity per subscriber $\left(\mathrm{C}_{\mathrm{T}}\right)$ can be expressed in terms of number of quantization levels $(\mathrm{Q})$, and the actual bandwidth for audio signal bandwidth (B. $W_{\mathrm{A}}$ ) for different interconnection network as [13]:

$$
\begin{aligned}
& C_{T \text { (Audio })}=B \cdot W_{A C} \log _{2} Q, \text { for }\left(\mathrm{OC}^{3} \mathrm{~N} \text { network }\right) \\
& C_{T(\text { Audio })}=B \cdot W_{A H} \log _{2} Q, \text { for }\left(\mathrm{OHC}^{2} \mathrm{~N} \text { network }\right)
\end{aligned}
$$

Where $\mathrm{B} . \mathrm{W}_{\mathrm{AC}}$ is the multiplication of the bandwidth of audio signal (around $3.4 \mathrm{KHz}-4 \mathrm{KHz}$ ) and the bisection bandwidth for optical crossbar connected cluster network $\left(\mathrm{OC}^{3} \mathrm{~N}\right)$, and also $\mathrm{B} . \mathrm{W}_{\mathrm{AH}}$ is the multiplication of the bandwidth of audio signal and the bisection bandwidth of optical hypercube connected cluster network $\left(\mathrm{OHC}^{2} \mathrm{~N}\right)$. In the same way, The transmission capacity per subscriber $\left(\mathrm{C}_{\mathrm{T}}\right)$ can be expressed in terms of number of quantization levels $(\mathrm{Q})$, and the actual bandwidth for audio signal bandwidth (B. $\left.\mathrm{W}_{\mathrm{A}}\right)$ for different interconnection network as follows [14]:

$$
\begin{aligned}
& C_{T \text { (Video })}=B \cdot W_{V C} \log _{2} Q, \text { for }\left(\mathrm{OC}^{3} \mathrm{~N} \text { network }\right) \\
& C_{T \text { (Video })}=B \cdot W_{V H} \log _{2} Q, \text { for }\left(\mathrm{OHC}^{2} \mathrm{~N} \text { network }\right)
\end{aligned}
$$

Where B. $\mathrm{W}_{\mathrm{VC}}$ is the multiplication of the bandwidth of video signal (around $6.8 \mathrm{MHz}-8 \mathrm{MHz}$ ) and the bisection bandwidth for optical crossbar connected cluster network $\left(\mathrm{OC}^{3} \mathrm{~N}\right)$, and also $\mathrm{B} \cdot \mathrm{W}_{\mathrm{VH}}$ is the multiplication of the bandwidth of video signal and the bisection bandwidth of optical hypercube connected cluster network $\left(\mathrm{OHC}^{2} \mathrm{~N}\right)$.

\section{SimULATION RESULTS AND DisCUSSIONS}

We have analyzed parametrically, and numerically the have been modeled, investigated and a analyzed numerically and parametrically over wide range of the affecting parameters optical interconnection networks are a promising design and performance parameters alternative for future systems. Numerous configurations with different degrees of optics, optoelectronics, network diameter, node degree, bisection width, scalability, transmission capacity, and cost evolution,. Based on the modeling equations analysis and the assumed set of the operating parameters as shown in Table 1 . 
Table 1: Proposed operating parameters for optical interconnection network model.

\begin{tabular}{|c|c|c|}
\hline $\begin{array}{c}\text { Operating } \\
\text { parameter }\end{array}$ & Definition & $\begin{array}{c}\text { Value and } \\
\text { units }\end{array}$ \\
\hline $\mathrm{B} . \mathrm{W}_{\mathrm{A}}$ & $\begin{array}{c}\text { Bandwidth of the } \\
\text { audio signal }\end{array}$ & $\begin{array}{c}3.4 \mathrm{KHz} \leq \\
\mathrm{B} . \mathrm{W}_{\mathrm{A}} \leq 4 \\
\mathrm{KHz}\end{array}$ \\
\hline $\mathrm{B} . \mathrm{W}_{\mathrm{V}}$ & $\begin{array}{c}\text { Bandwidth of the } \\
\text { video signal }\end{array}$ & $\begin{array}{c}6.8 \mathrm{MHz} \leq \\
\mathrm{B} . \mathrm{W}_{\mathrm{V}} \leq 8 \\
\mathrm{MHz}\end{array}$ \\
\hline $\mathrm{Q}$ & $\begin{array}{c}\text { Number of } \\
\text { quantization levels }\end{array}$ & $16 \leq \mathrm{Q} \leq$ \\
1024
\end{tabular}

The following facts are assured as shown in the series of Figs. (4-29):

i) As shown in Fig. 4 has indicated that as both number of clusters and number of processors per cluster increase this lead to increase in total number of nodes or processors in the case of optical crossbar connected cluster network.

ii) Fig. 5 has assured that as both number of processors per cluster and network diameter increase this lead to increase in total number of nodes or processors in the case of optical hypercube connected cluster network.

iii) As shown in Fig. 6 has indicated that as both number of processors and number of processors per cluster increase this lead to increase in node degree or link complexity in the case of optical crossbar connected cluster network.

iv) Fig. 7 has assured that as network diameter increase this leads to increase in node degree or link complexity the case of optical hypercube connected cluster network.

v) Figs. $(8,9)$ have demonstrated that as total number of processors increase and number of processors per cluster decrease this lead to increase in number of links as in the case of both optical crossbar connected cluster and optical hypercube connected cluster networks. We observed that number of links in optical crossbar connected cluster network is larger than optical hypercube connected cluster network.

vi) As shown in Fig. 10 has proved that as total number of processors increase, this result in increasing bisection bandwidth in both optical crossbar connected cluster and optical hypercube connected cluster networks.

vii) As shown in Fig. 11 has demonstrated that as both number of processors per clusters and total number of processors increase, this result in increasing average message distance in the case of optical hypercube connected cluster network but average message distance equal to unity in the case of optical crossbar connected cluster network.

viii) Figs. (12-21) have assured that as number of processors per cluster, number of tunable VCSELs, number of detectors, number of waveguides, number of demultiplexers, and number of clusters increase this result in increasing in network cost in the case of both optical crossbar connected cluster and optical hypercube connected cluster networks. We have indicated that optical crossbar connected cluster network is higher network cost than optical hypercube connected cluster network.

ix) As shown in Figs. (22-25) have indicated that as number of quantization levels, number of processors and bandwidth of audio signal increase, this lead to increase in the transmission capacity of bit rates for both crossbar connected cluster and optical hypercube connected cluster networks. We have observed that optical crossbar connected cluster network is higher transmission bit rates than optical hypercube connected cluster network.

x) As shown in Figs. (26-29) have indicated that as number of quantization levels, number of processors and bandwidth of video signal increase, this lead to increase in the transmission capacity of bit rates for both crossbar connected cluster and optical hypercube connected cluster networks. We have observed that optical crossbar connected cluster network is higher transmission bit rates than optical hypercube connected cluster network. 


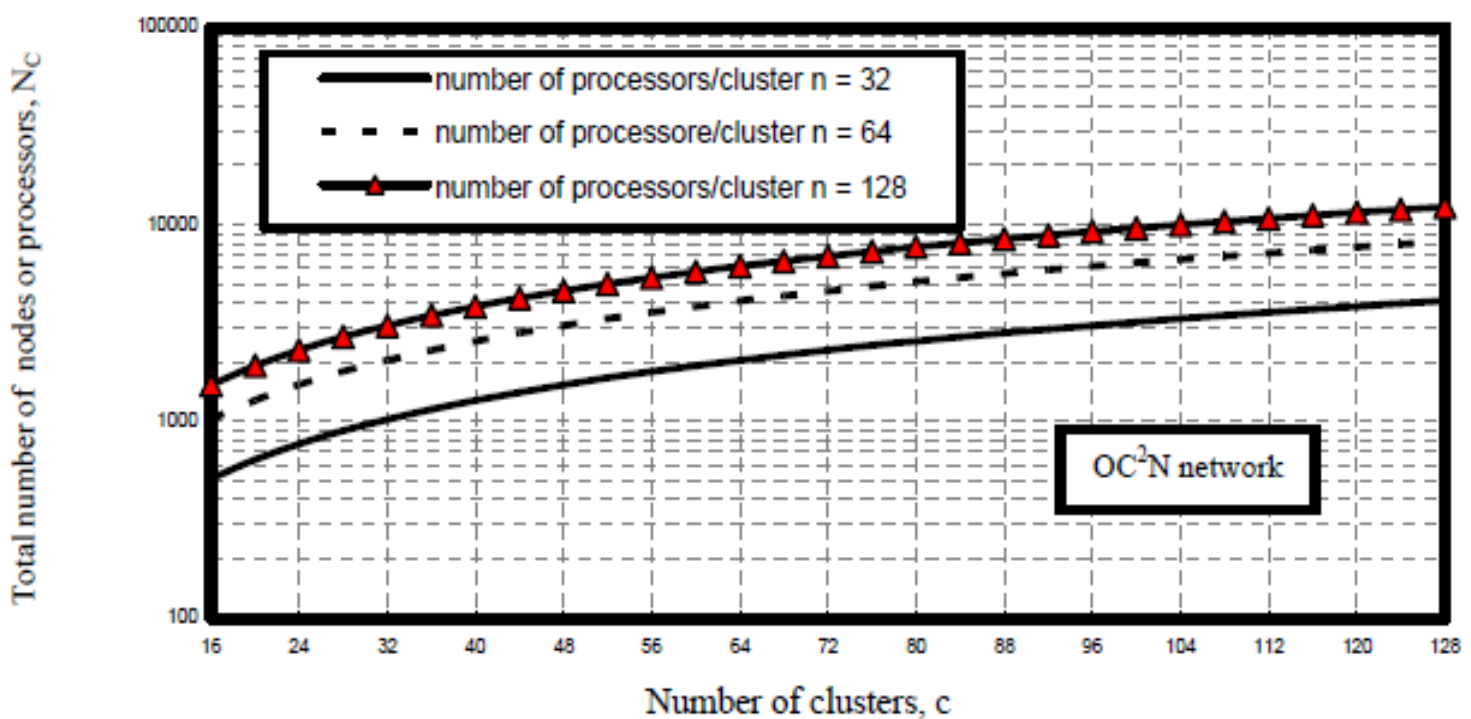

Fig. 4. Variations of total number of processors against number of clusters at the assumed set of parameters.
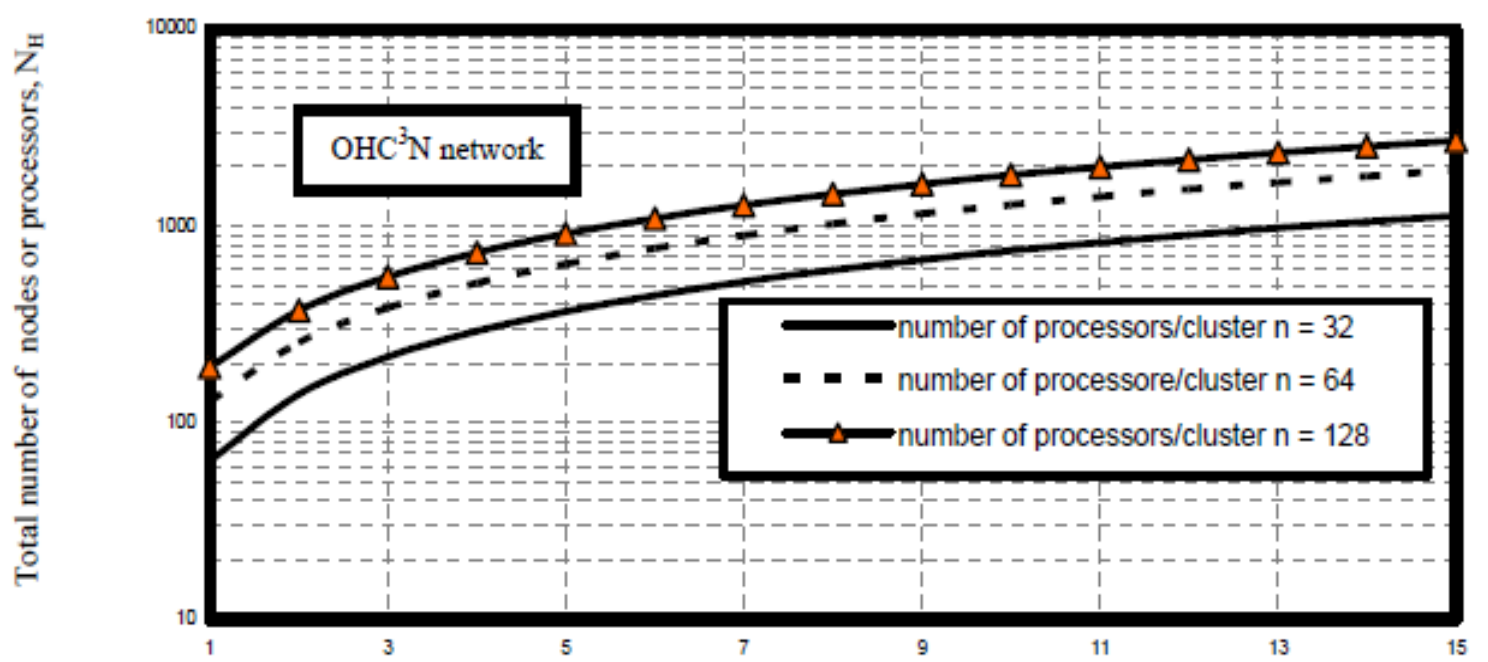

Network diameter, $\mathrm{d}$

Fig. 5. Variations of total number of processors against network diameter at the assumed set of parameters.

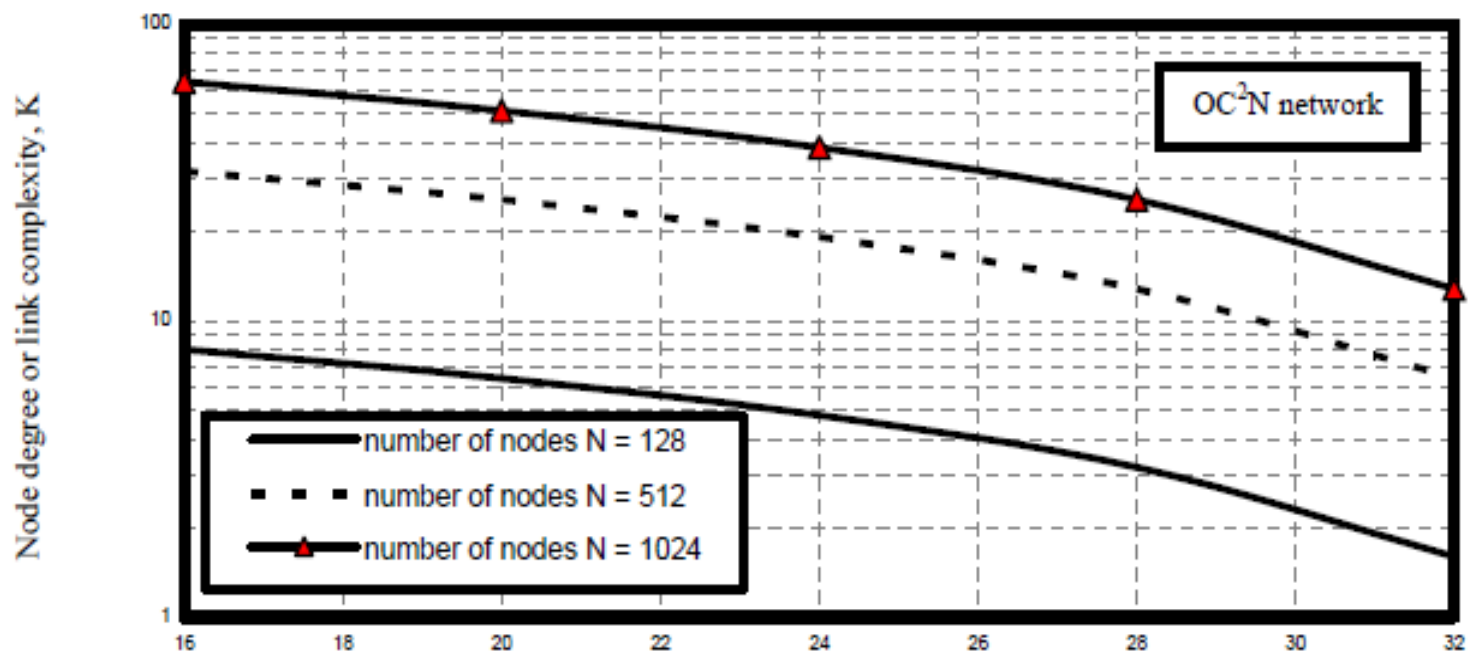

Number of processors per cluster, $n$

Fig. 6. Variations of node degree or link complexity against number of processors/cluster at the assumed set of parameters. 


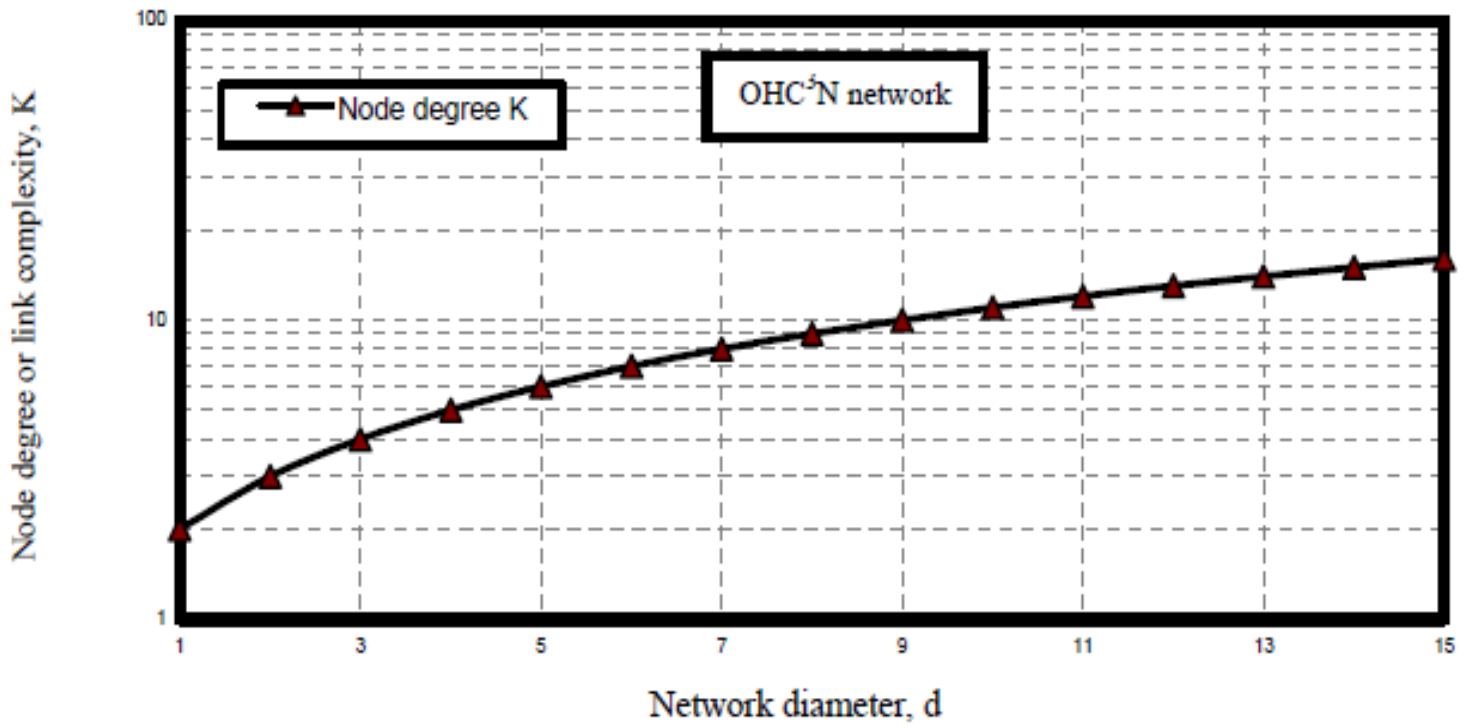

Fig. 7. Variations of node degree or link complexity against network diameter at the assumed set of parameters.

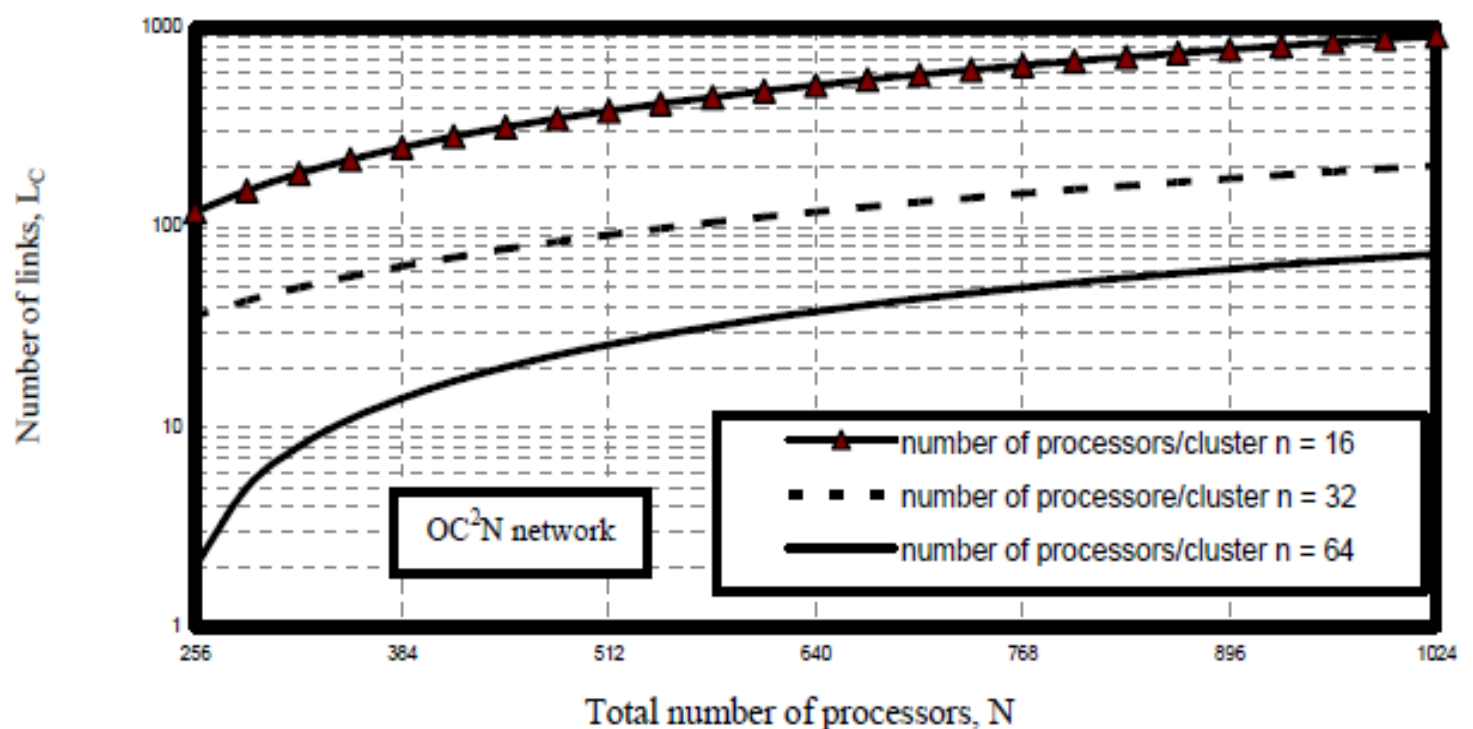

Fig. 8. Variations of number of links against total number of processors at the assumed set of parameters.

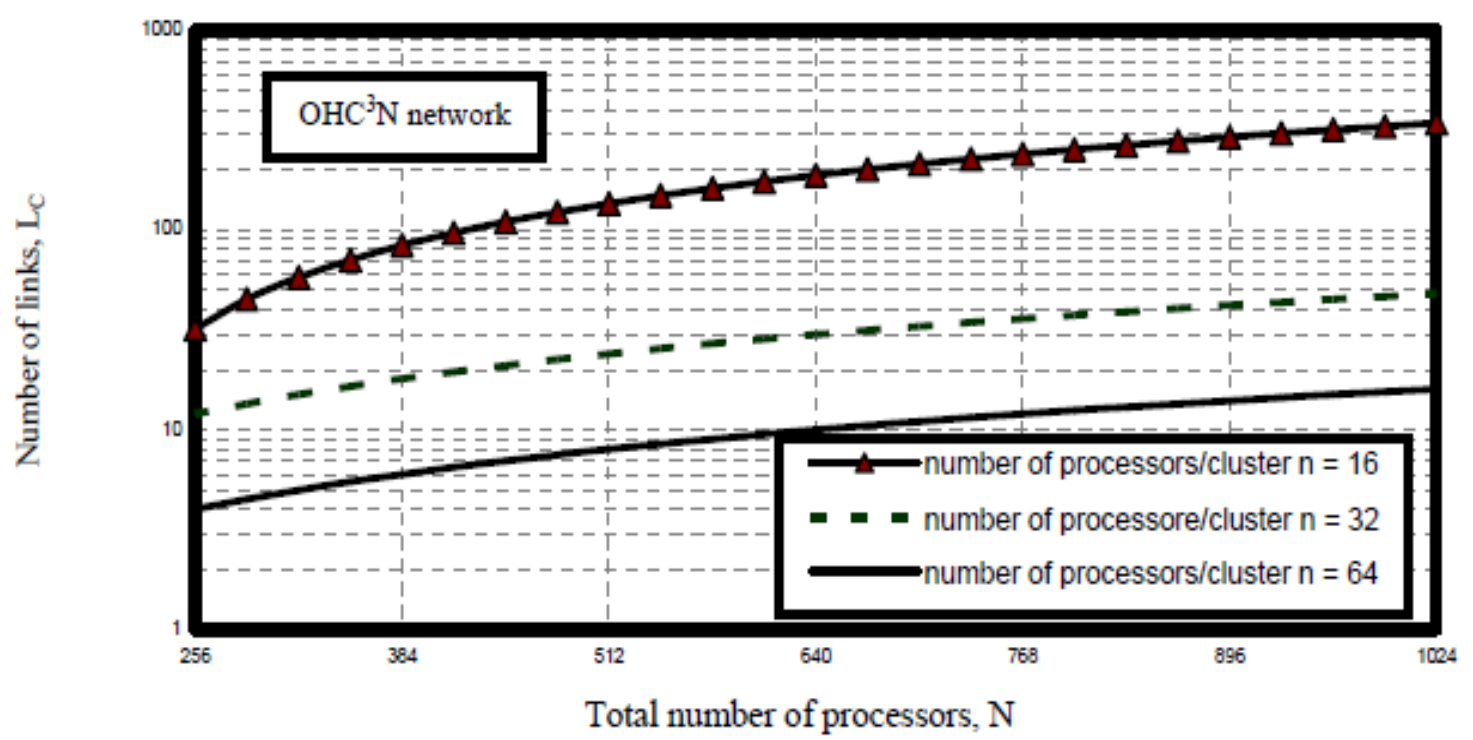

Fig. 9. Variations of number of links against total number of processors at the assumed set of parameters. 


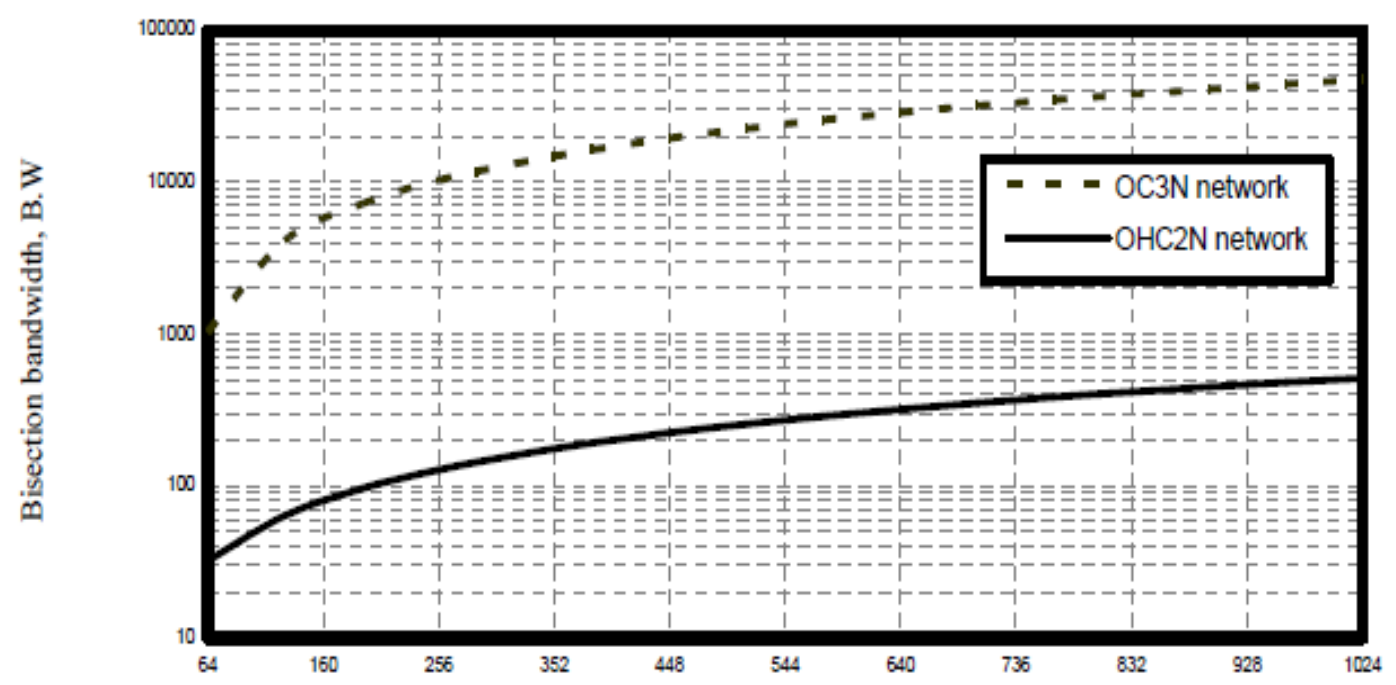

Total number of processors, $\mathrm{N}$

Fig. 10. Variations of number of links against total number of processors at the assumed set of parameters.

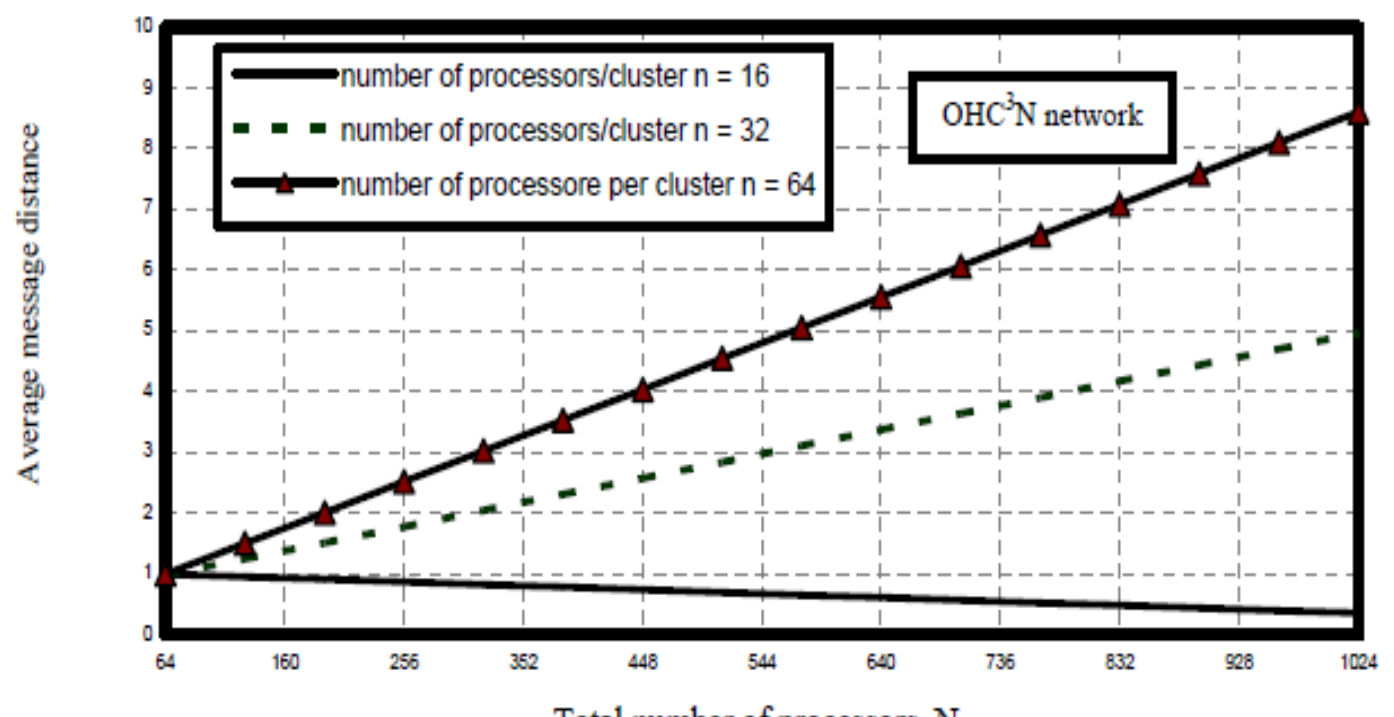

Total number of processors, $\mathrm{N}$

Fig. 11. Variations of average message distance against total number of processors at the assumed set of parameters.

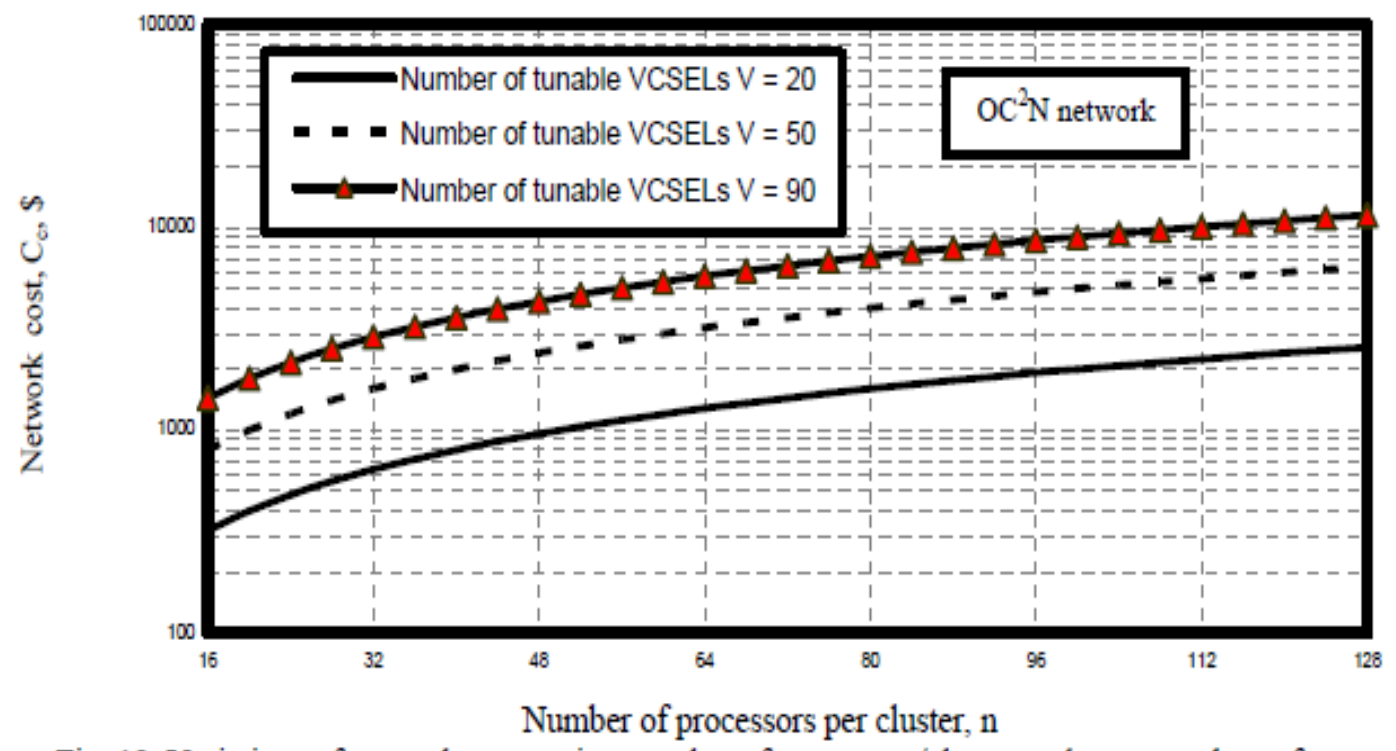

Fig. 12. Variations of network cost against number of processors/cluster at the assumed set of parameters. 


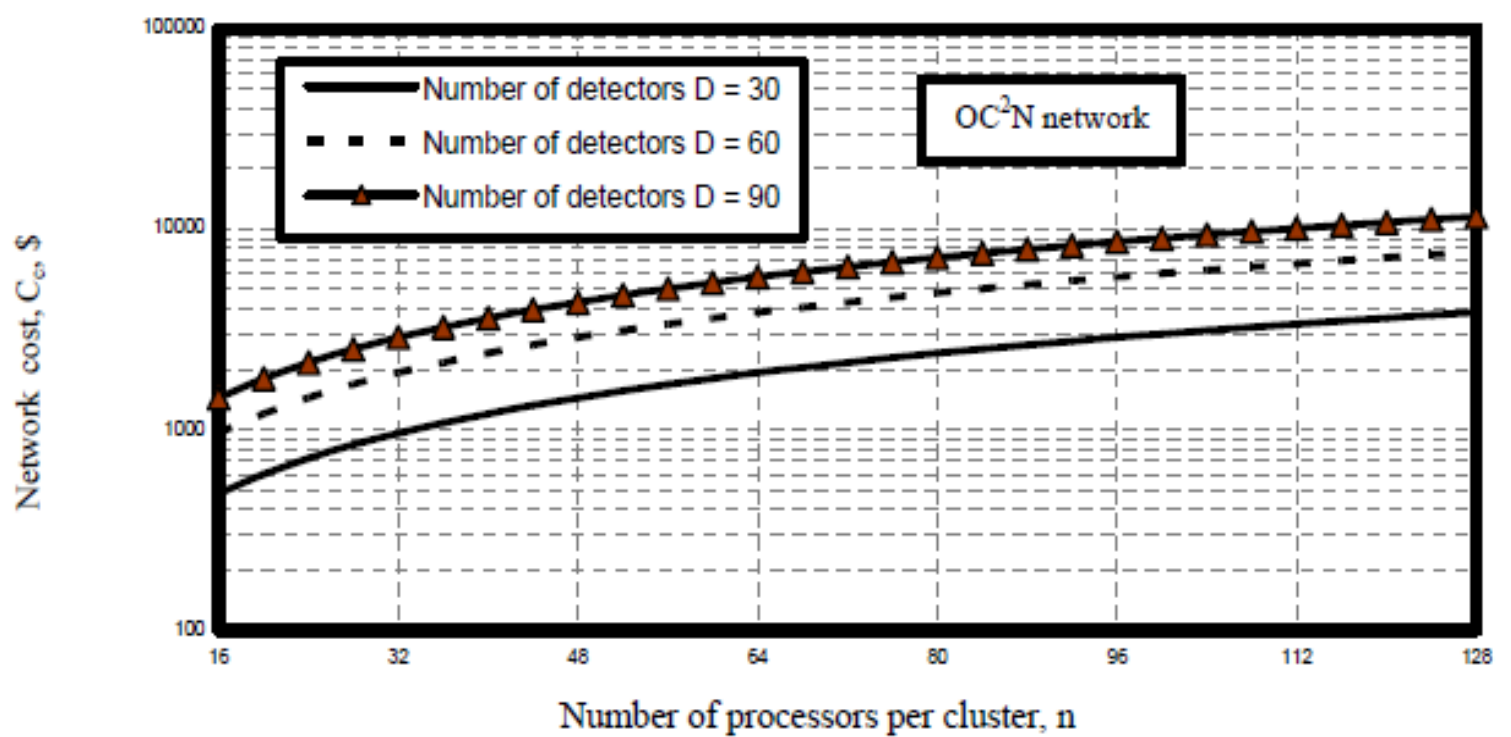

Fig. 13. Variations of network cost against number of processors/cluster at the assumed set of parameters.

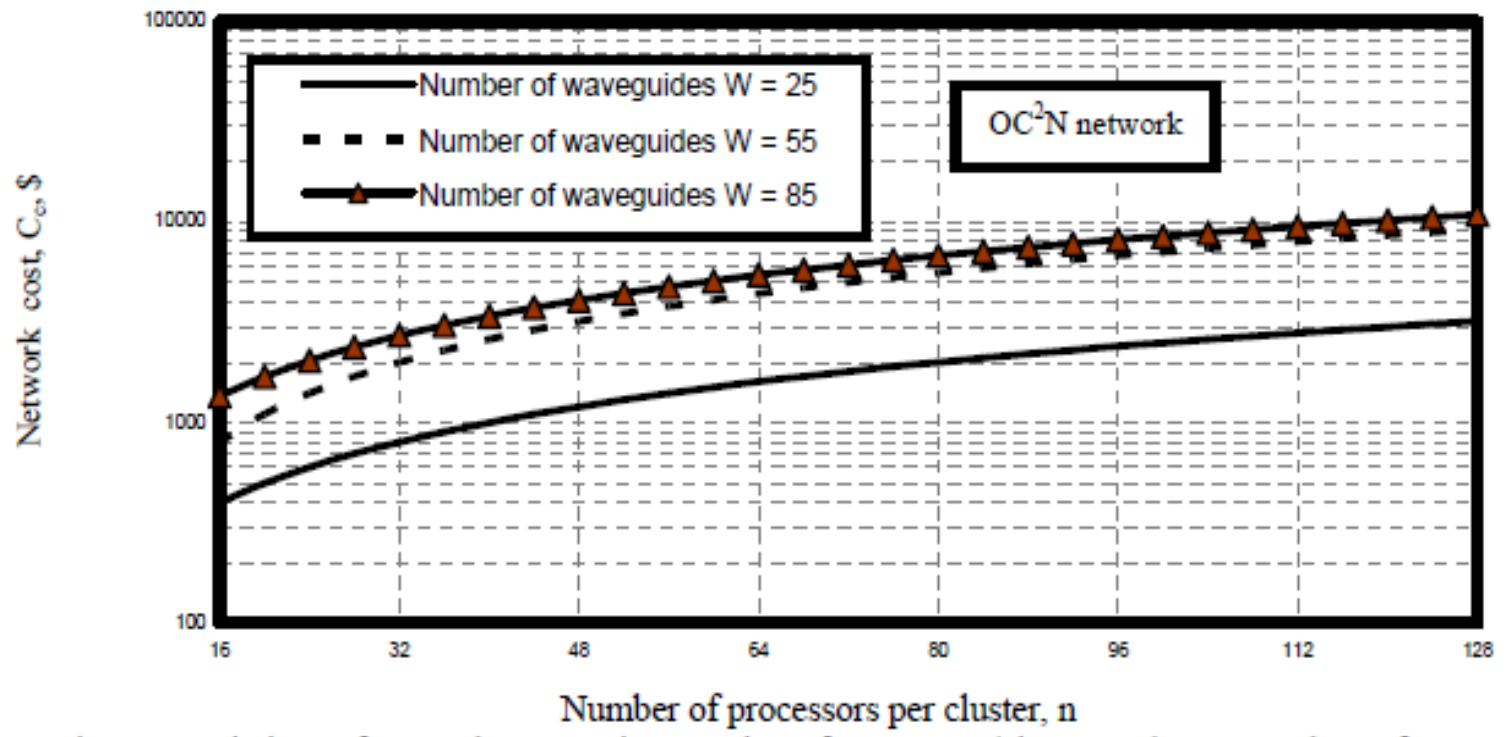

Fig. 14. Variations of network cost against number of processors/cluster at the assumed set of parameters.

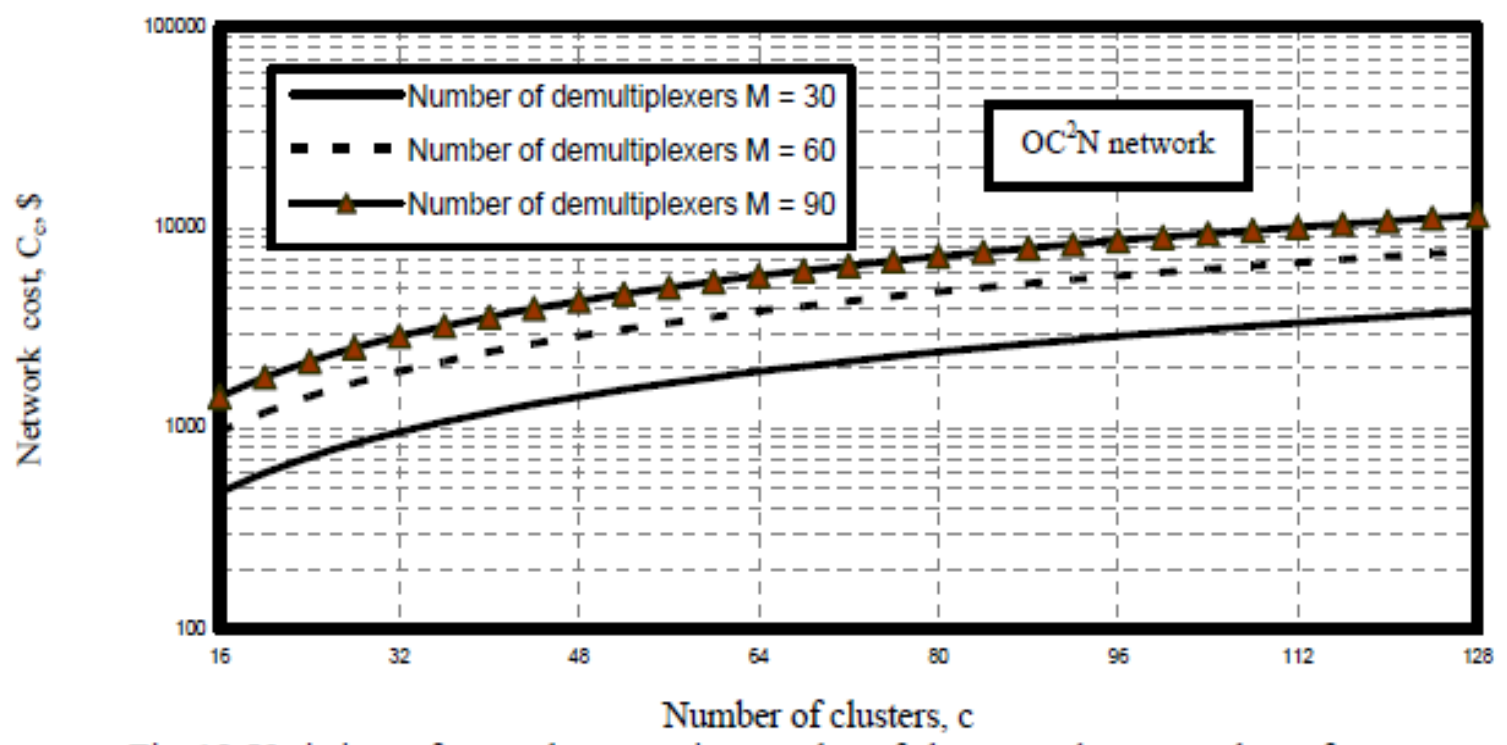

Fig. 15. Variations of network cost against number of clusters at the assumed set of parameters. 


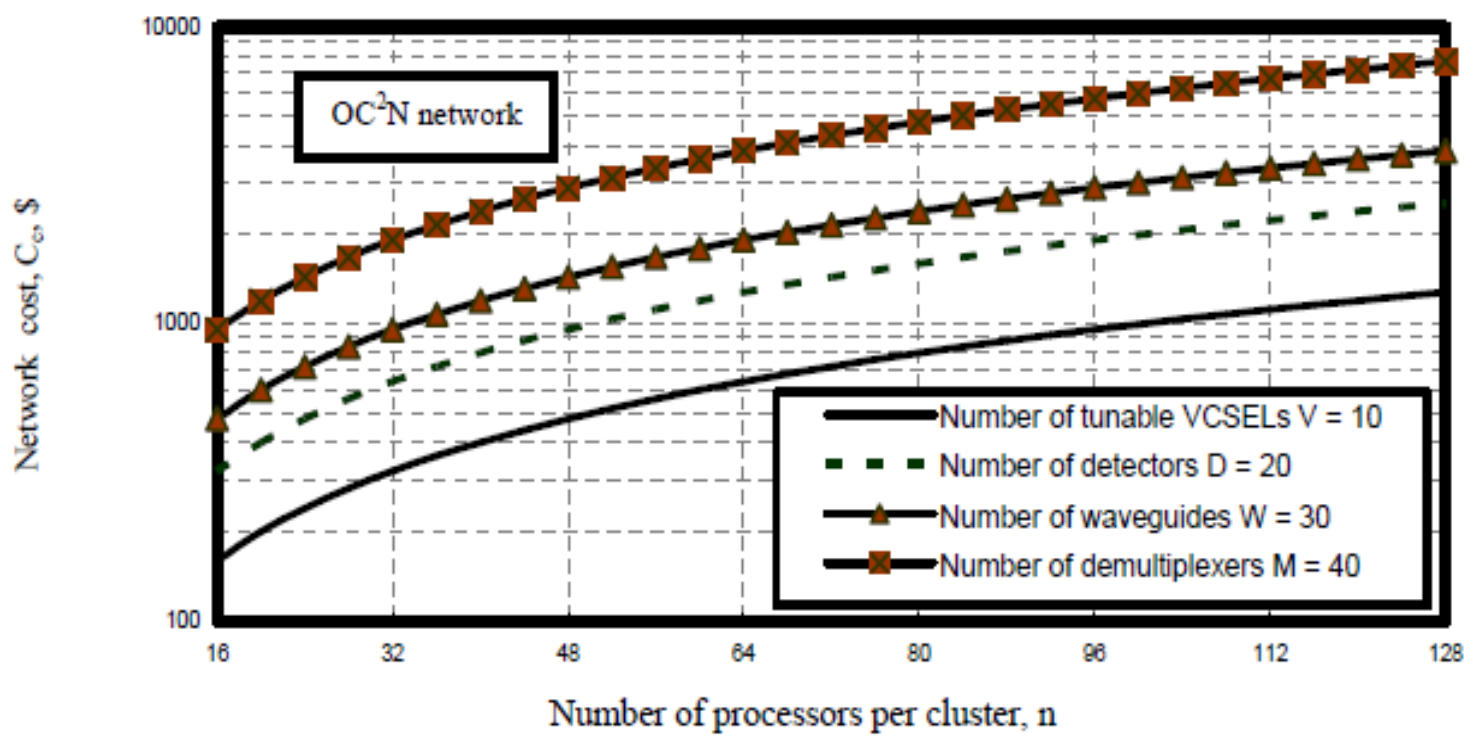

Fig. 16. Variations of network cost against number of processors/cluster at the assumed set of parameters.

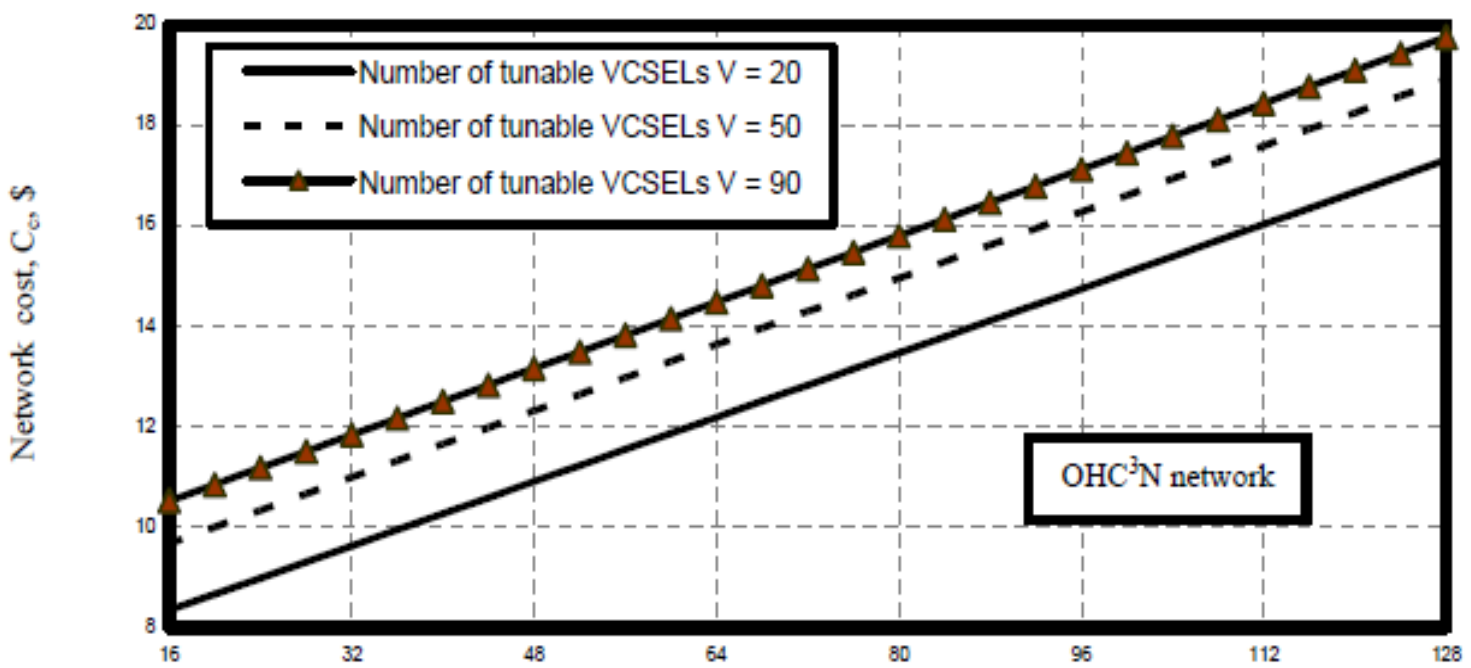

Number of processors per cluster, $n$

Fig. 17. Variations of network cost against number of processors/cluster at the assumed set of parameters.

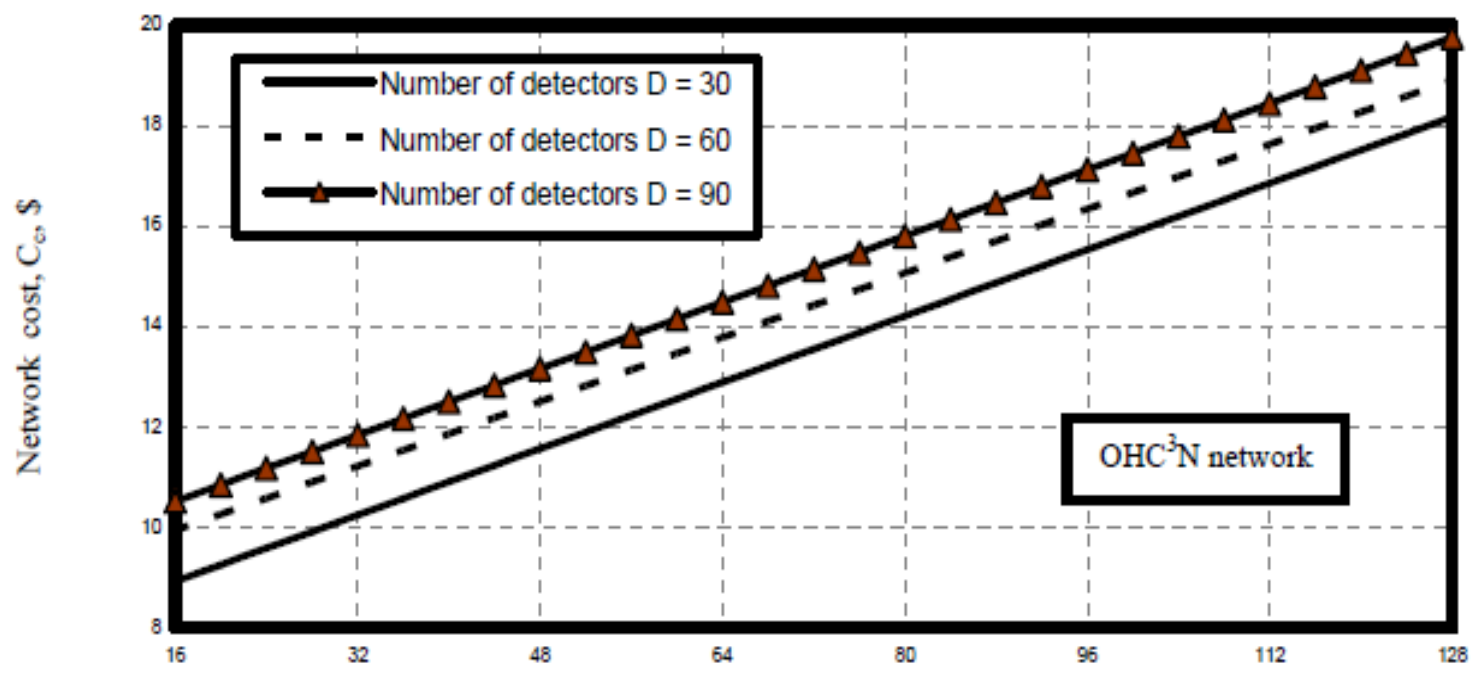

Number of processors per cluster, $n$

Fig. 18. Variations of network cost against number of processors/cluster at the assumed set of parameters. 


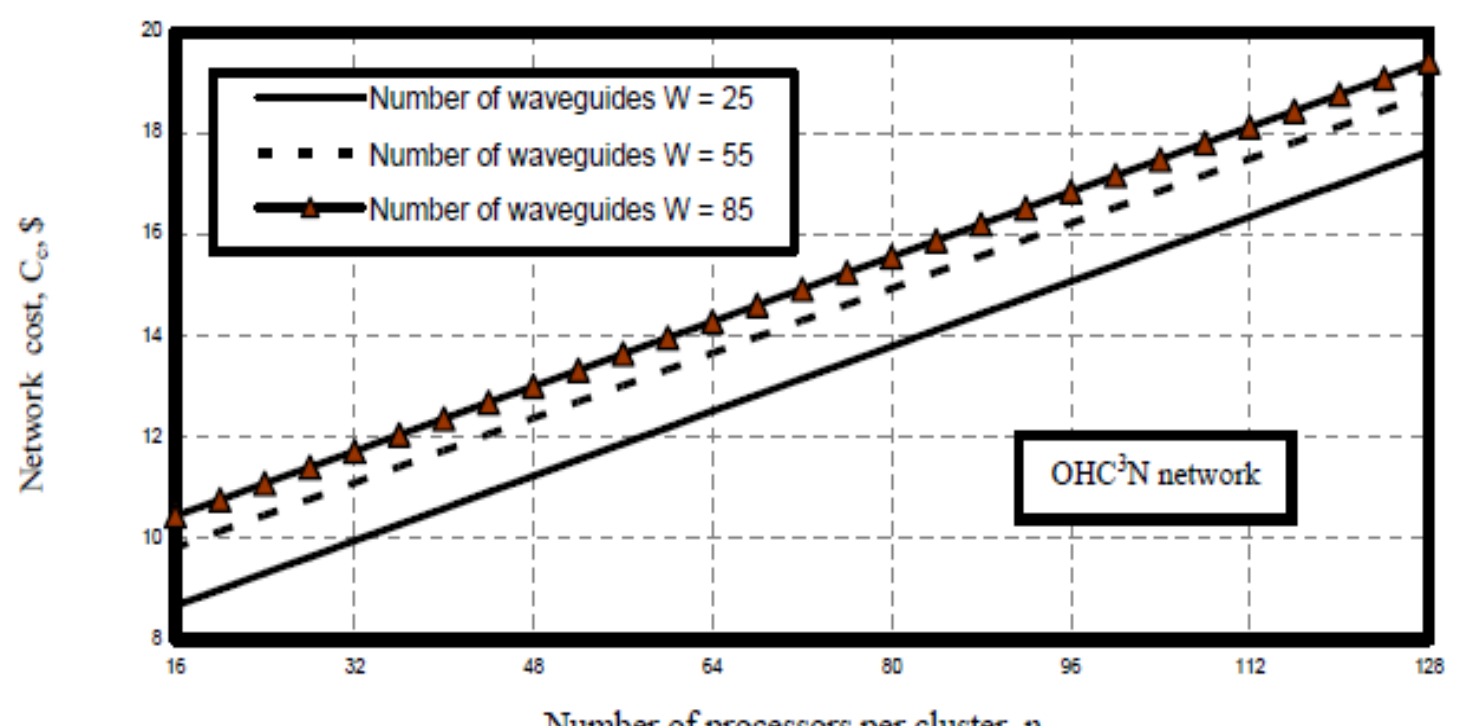

Fig. 19. Variations of network cost against number of processors/cluster at the assumed set of parameters.

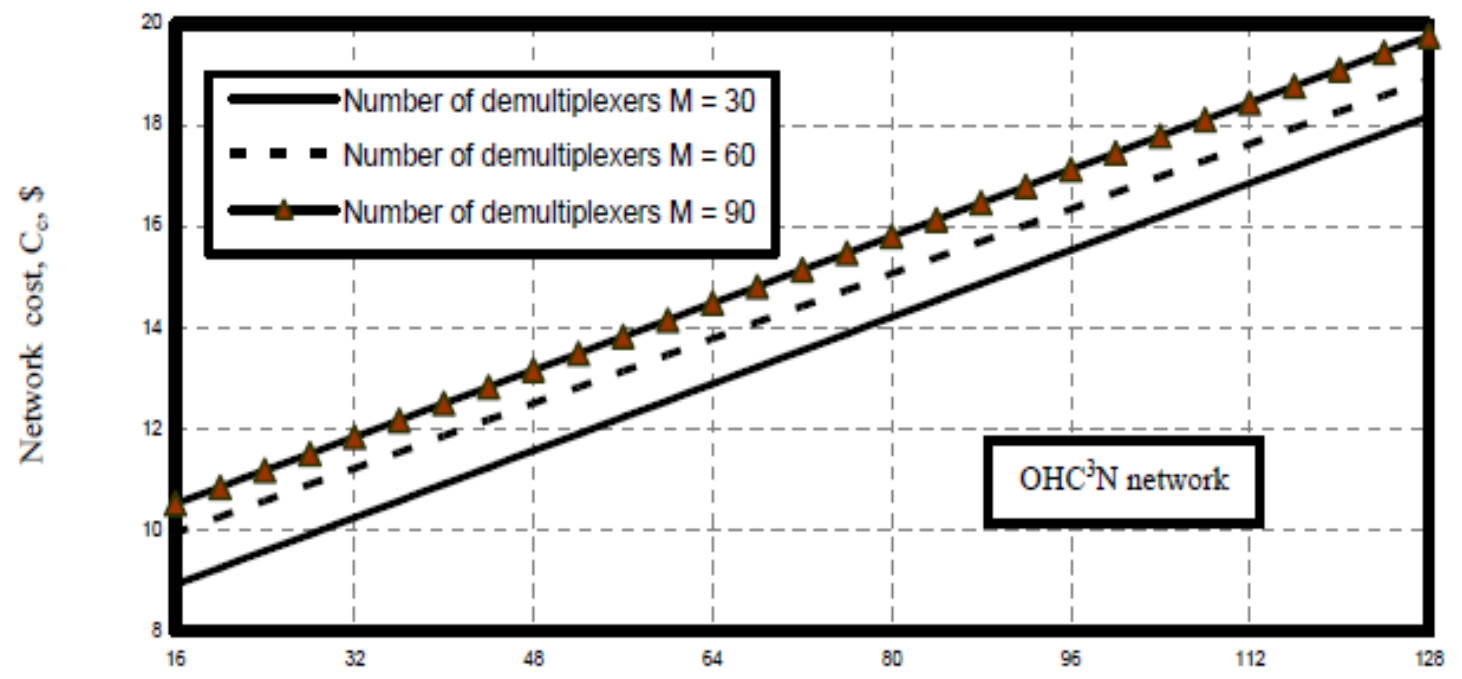

Number of clusters, c

Fig. 20. Variations of network cost against number of clusters at the assumed set of parameters.

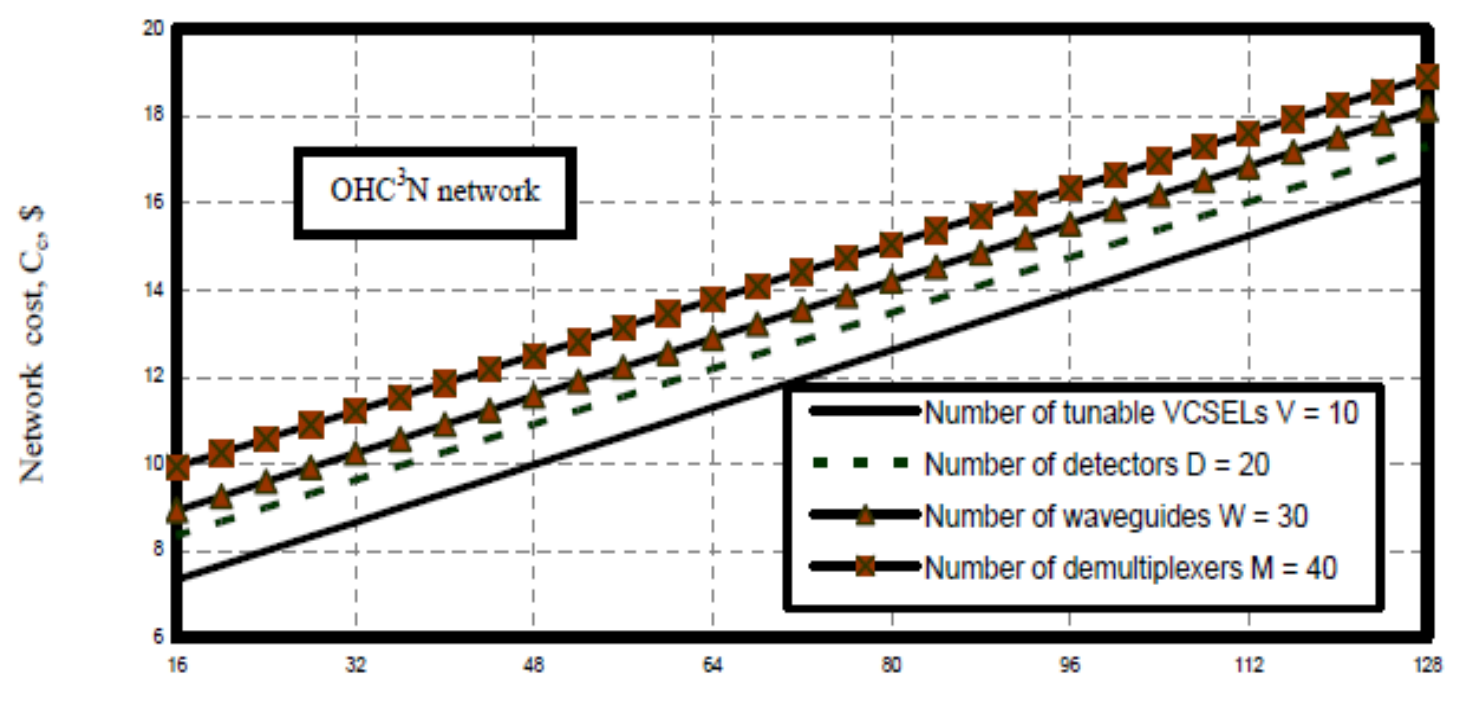

Number of processors per cluster, $n$

Fig. 21. Variations of network cost against number of processors/cluster at the assumed set of parameters. 


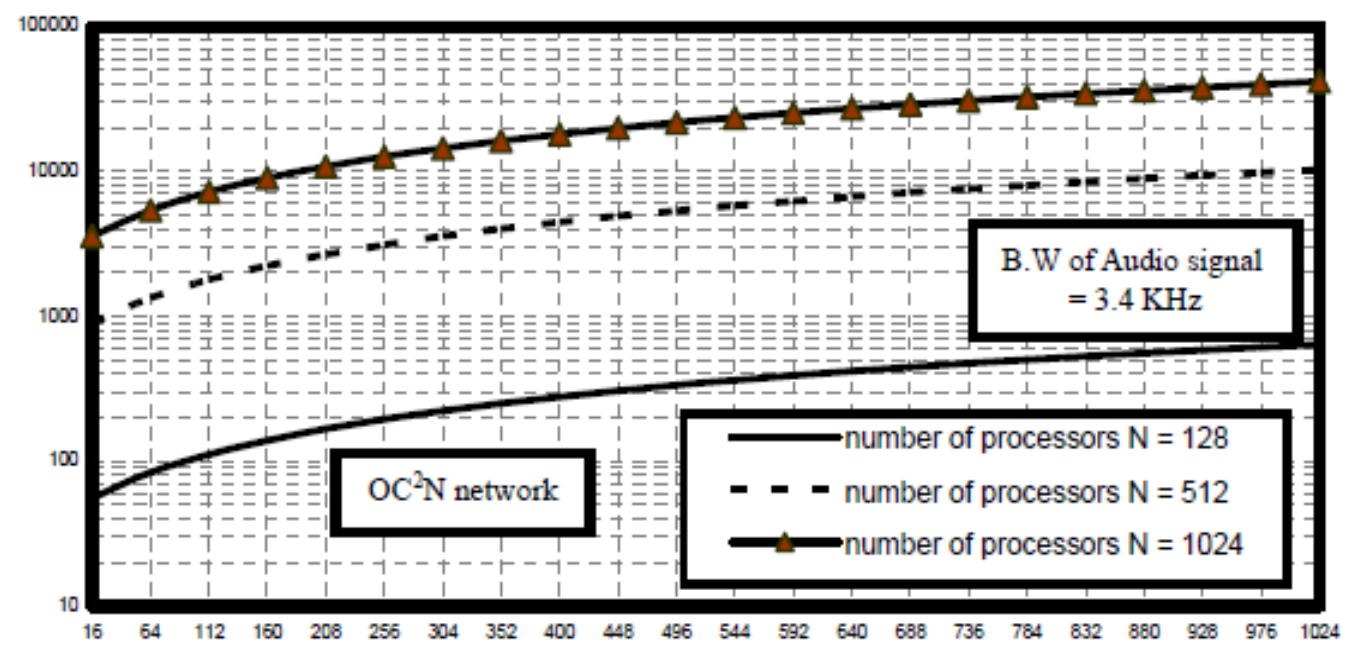

Number of quantization levels, $Q$

Fig. 22. Variations of the capacity of audio signal versus number of quantization levels at the assumed set of parameters.
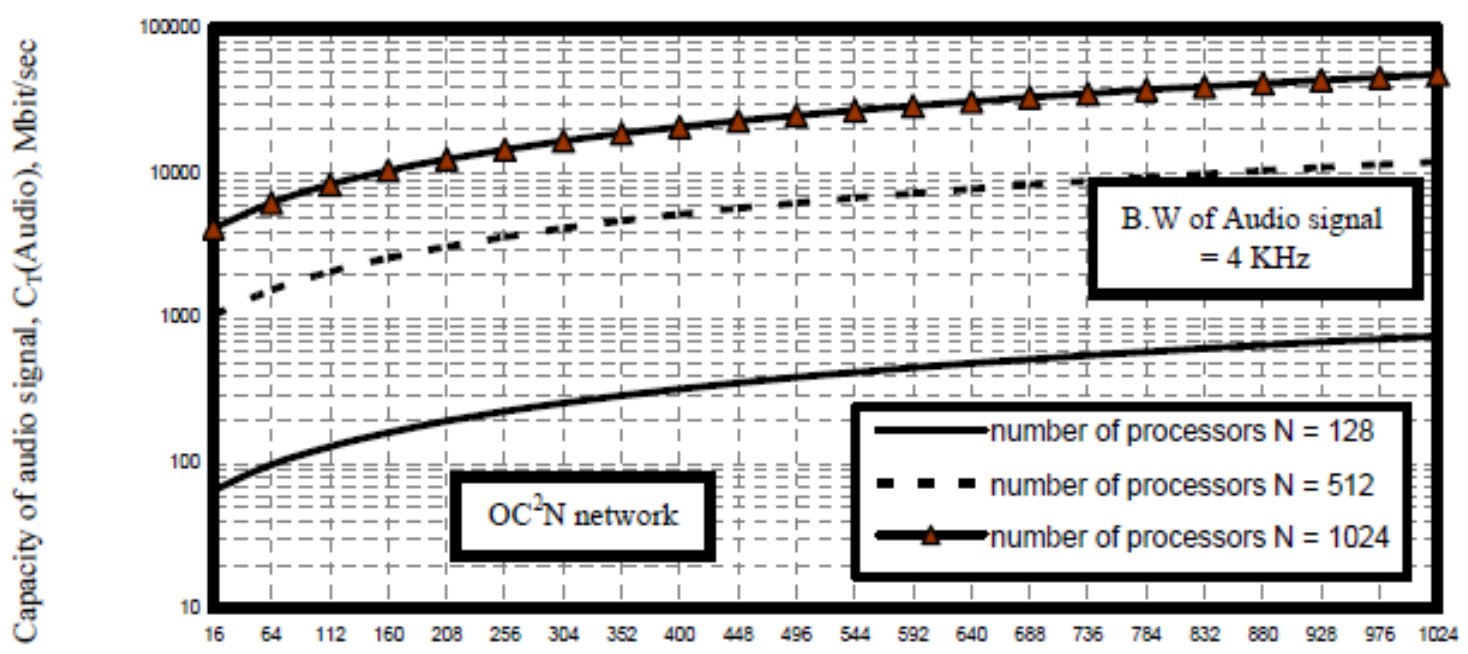

Number of quantization levels, $Q$

Fig. 23. Variations of the capacity of audio signal versus number of quantization levels at the assumed set of parameters.
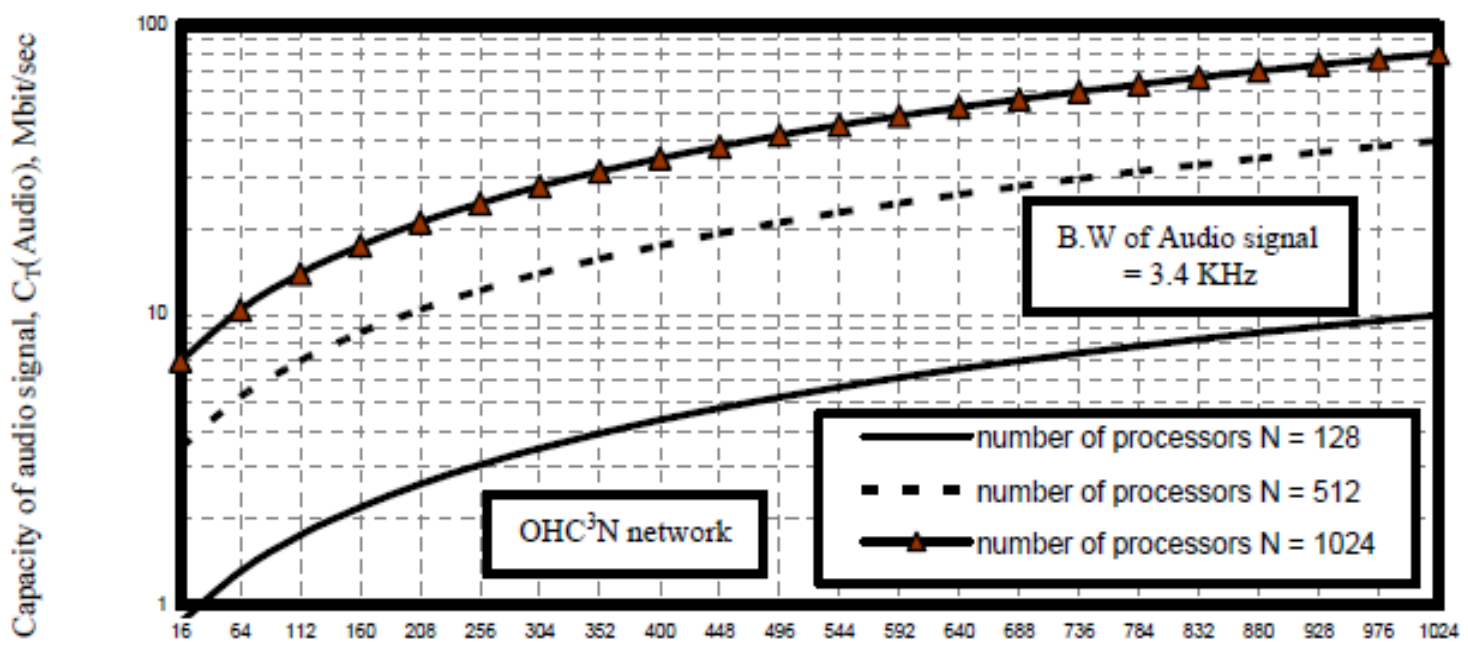

Number of quantization levels, $Q$

Fig. 24. Variations of the capacity of audio signal versus number of quantization levels at the assumed set of parameters. 


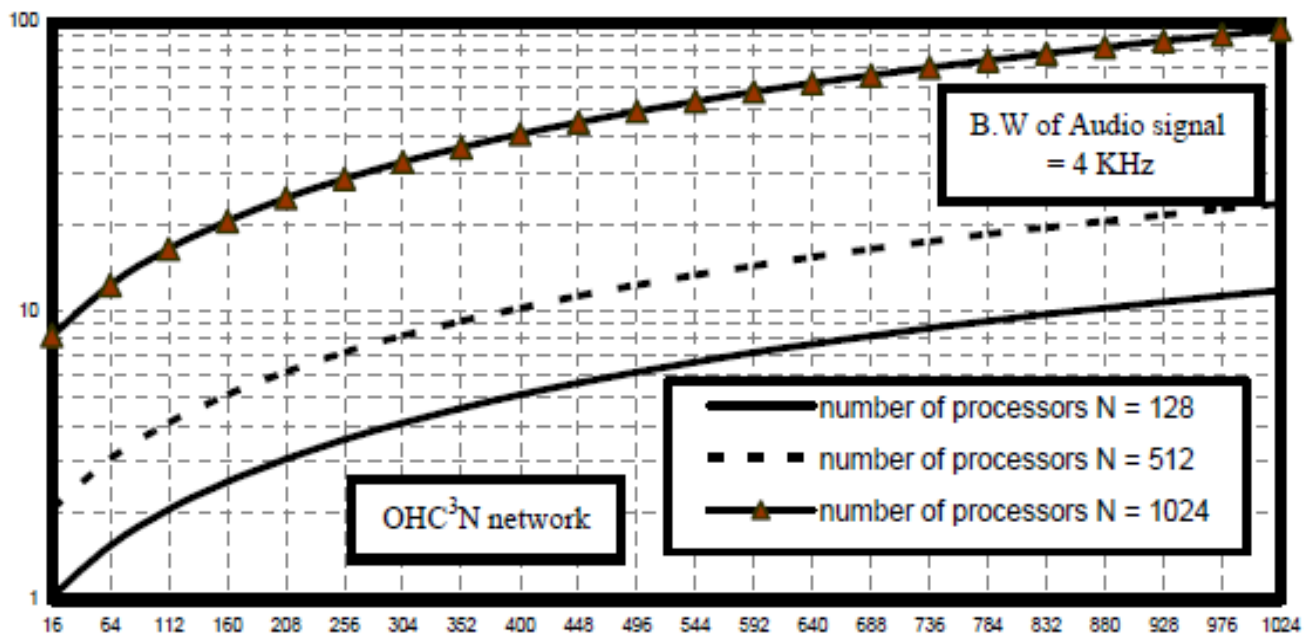

Number of quantization levels, $Q$

Fig. 25. Variations of the capacity of audio signal versus number of quantization levels at the assumed set of parameters.

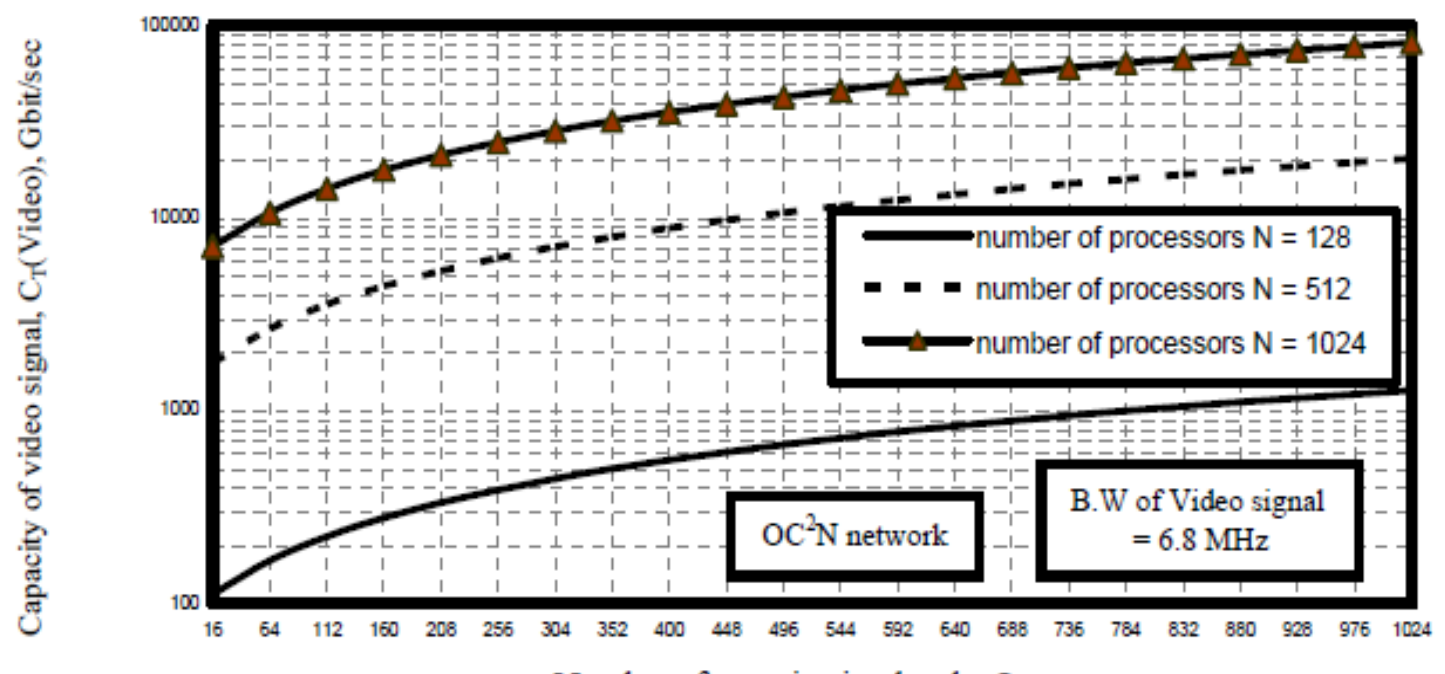

Number of quantization levels, $Q$

Fig. 26. Variations of the capacity of audio signal versus number of quantization levels at the assumed set of parameters.

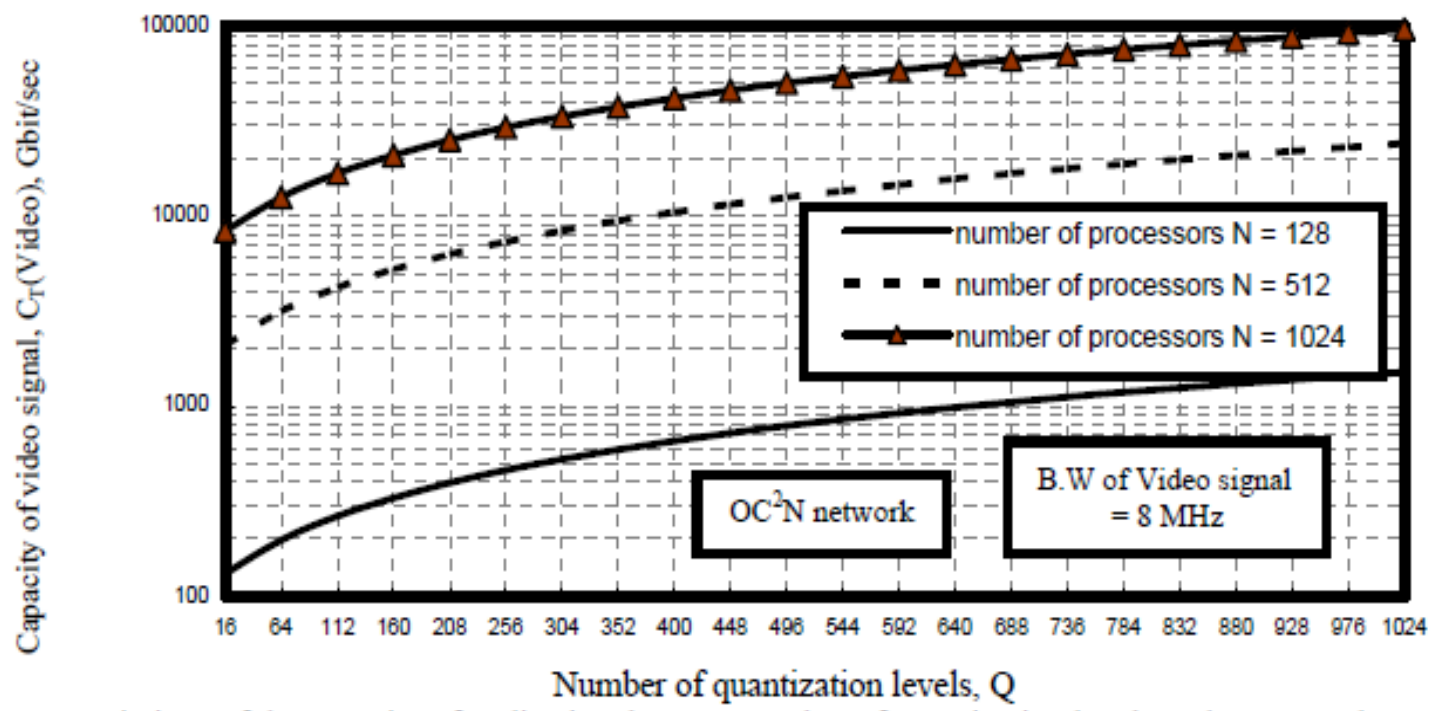

Fig. 27. Variations of the capacity of audio signal versus number of quantization levels at the assumed set of parameters. 


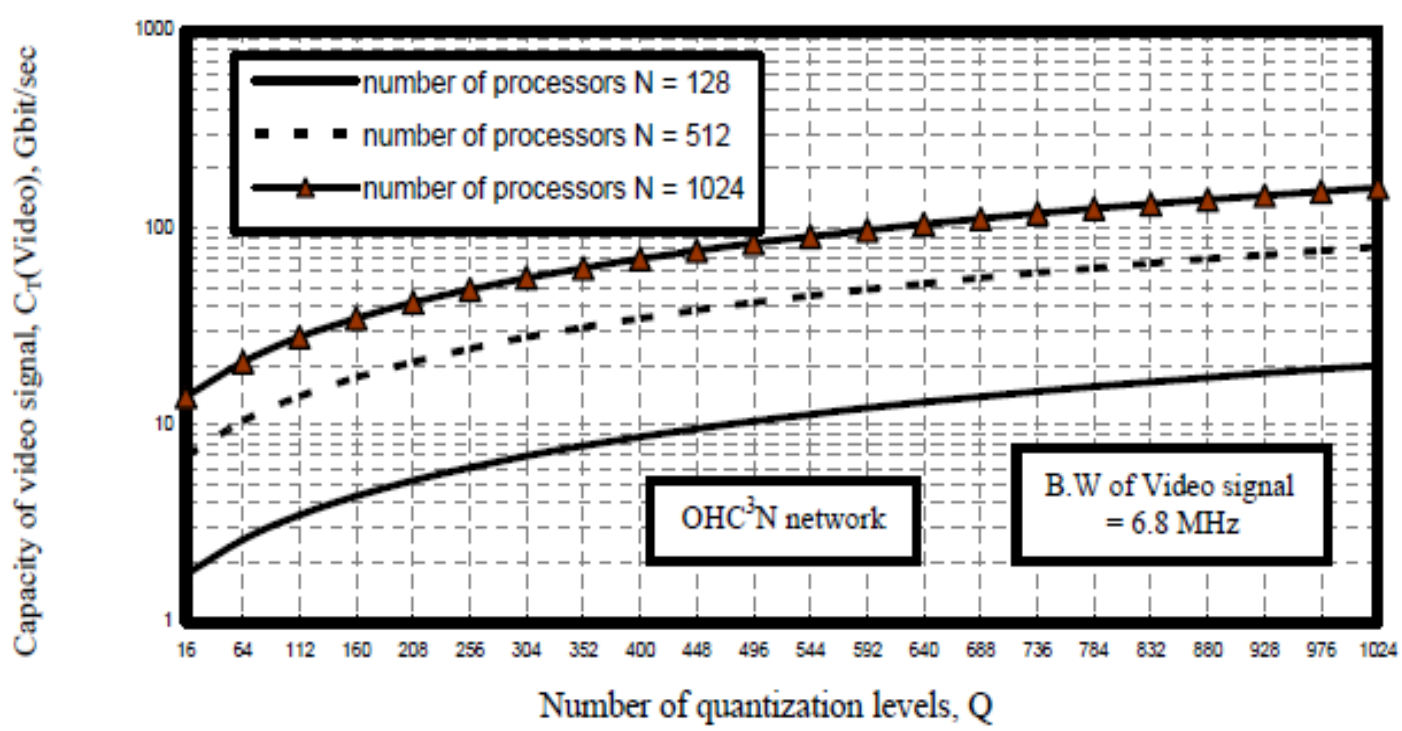

Fig. 28. Variations of the capacity of audio signal versus number of quantization levels at the assumed set of parameters.

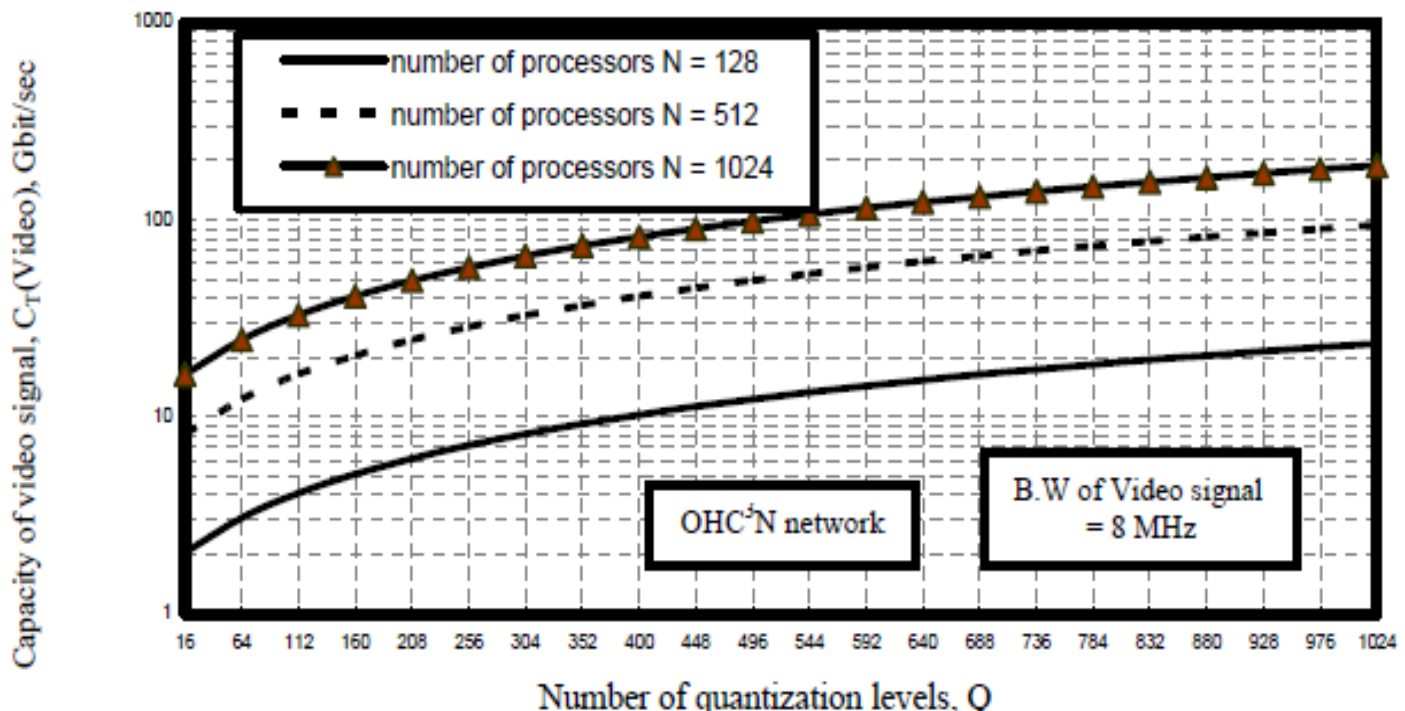

Fig. 29. Variations of the capacity of audio signal versus number of quantization levels at the assumed set of parameters.

\section{CONCLUSIONS}

In a summary, we have presented the large scale optical interconnection systems compared with the small and medium scalability of these systems in different optical connection networks. It is evident that the increased number of clusters and number of processors per cluster, the increased total number of processors and number of links for both types of optical interconnection networks. As well as the increased number of tunable VCSELs, number of detectors, number of waveguides, and number of demultiplexers, this result in the increased additional costs to both optical interconnection networks. Moreover the increased number of quantization levels, number of processors, an bandwidth of audio and video signals this lead to the increased transmission bit rate capacity for both optical interconnection networks. It is evident that optical crossbar connected cluster network is higher node degree, link complexity, bisection bandwidth, number of links, number of processors, transmission bit rate capacity for audio and video signals, and higher additional network costs than optical hypercube connected cluster network. We have summarized the complete comparison of large scale of our optical interconnection systems with their medium optical interconnection systems [15]. 
Table 2: Comparison Our large scale optical interconnection system with Simulation results as in Refs. [13-15].

\begin{tabular}{|c|c|c|c|c|}
\hline & \multicolumn{2}{|c|}{$\begin{array}{l}\text { Our large scale optical interconnection } \\
\text { systems }\end{array}$} & \multicolumn{2}{|c|}{$\begin{array}{l}\text { Simulation results for medium scale optical } \\
\text { interconnection systems as in Refs. [13-15] }\end{array}$} \\
\hline & \multicolumn{4}{|c|}{ Optical Interconnection Networks } \\
\hline & \multicolumn{2}{|c|}{ Our simulation results for large scale } & \multicolumn{2}{|c|}{ Their simulation results for medium scale } \\
\hline $\begin{array}{l}\text { Type of Optical Interconnection } \\
\text { Networks }\end{array}$ & $\mathrm{OC}^{3} \mathrm{~N}$ network & OHC ${ }^{2} \mathbf{N}$ network & OC $^{3} \mathrm{~N}$ network & OHC ${ }^{2} \mathrm{~N}$ network \\
\hline Number of processors/cluster & 128 & 128 & 16 & 16 \\
\hline Total number of processors & 16384 & 1920 & 256 & 240 \\
\hline Node degree or link complexity & 64 & 16 & 16 & 10 \\
\hline Number of links & 912 & 344 & 120 & 96 \\
\hline Additional Network Costs & $15540 \$$ & $51 \$$ & $972 \$$ & $4 \$$ \\
\hline Transmission capacity of audio signal & $48.24 \mathrm{Gbit} / \mathrm{sec}$ & $88 \mathrm{Mbit} / \mathrm{sec}$ & $800 \mathrm{Mbit} / \mathrm{sec}$ & $6 \mathrm{Mbit} / \mathrm{sec}$ \\
\hline Transmission capacity of video signal & $96.46 \mathrm{Tbit} / \mathrm{sec}$ & $196 \mathrm{Gbit} / \mathrm{sec}$ & $30 \mathrm{Gbit} / \mathrm{sec}$ & $13 \mathrm{Gbit} / \mathrm{sec}$ \\
\hline
\end{tabular}

It is clear from the comparison the best performance of our large scale optical interconnection systems in large number of processors and links and transmission bit rate capacity of audio and video signals in both types of optical interconnection networks over medium scale optical interconnection systems as mentioned in the previous studies. But our results presents additional higher network costs and complexity with large optical interconnection scalability compared with medium scalability of optical interconnection systems as mentioned in the previous studies.

\section{REFERENCES}

[1] A. Pattavina, M. Martinelli, G. Maier, and P. Boffi, "Techniques and Technologies Towards All Optical Switching," Optical Networks Magazine, Vol. 1, No. 2, pp. 75-93, Apr. 2000.

[2] E. Griese, "A high Performance Hybrid Electrical Optical Interconnection Technology for High Speed Electronic Systems," IEEE Transactions On Advanced Packaging, Vol. 24, No. 3, pp. 375-383, Aug. 2001.

[3] D. V. Plant, M. B. Venditti, E. Laprise, J. Faucher, K. Razavi, M. Châteaunuef, A. G. Kirk, and J. S. Ahearn, "256 Channel Bidirectional Optical Interconnect Using VCSELs and Photodiodes on CMOS," IEEE Journal of Lightwave Technology, Vol. 19, No. 8, pp. 1093-1103, Aug. 2001.

[4] P. M. Hagelin, U. Krishnamoorthy, J. P. Heritage, and O. Solgaard, "Scalable Optical Cross Connect Switch Using Micromachined Mirrors," IEEE Photonics Technology Letters, Vol. 12, No. 7, pp. 882-884, July 2000.

[5] H. H. Abadi, and S. Azad, "An Empirical Comparison of OTIS Mesh and OTIS Hypercube Multicomputer Systems under Deterministic Routing", Proceedings of the $19^{\text {th }}$ IEEE International Parallel and
Distributed Processing Symposium (IPDPS'05), Vol. 8, No. 4, pp. 4-8, 2005.

[6] N. G. Kini , M. S. Kumar and B. S. Satyanarayana, "Enhancement of Speed in Massively Parallel Computers using Optical Technology", Second National Conference on Frontier Research Areas in Computing Sciences (Proceedings), Department of Computer Science, Bharathidasan University, Tiruchirapalli, Tamilnadu, India, Oct. 2007.

[7] N. G. Kini , M. S. Kumar and B. S. Satyanarayana, "Free Space Optical Interconnection Networks for High Performance Parallel Computing Systems", IEEE Sponsored International conference on Recent Applications of Soft Computing in Engineering \& Technology RASIET-07, Institute of Engineering \& Technology, Alwar, Rajasthan, India, pp. 134-137, Dec. 2007.

[8] Z. Li, Y. Zhang, Y. Chen and R. Tang, "Design and Implementation of High Performance Interconnection Network," Proceedings of the Fourth International Conference on Parallel and Distributed Computing, Applications and Technologies, PDCAT'2003, pp. 27-29, Aug. 2003.

[9] N. G. Kini, M. S. Kumar and H. S. Mruthyunjaya, "Analysis and Comparison of Torus Embedded Hypercube Scalable Interconnection Network for Parallel Architecture," International journal of Computer Science and Network Security, vol. 9, No.1, pp. 242-247, Jan. 2009.

[10] N. G. Kini, M. S. Kumar and S. H. Mruthyunjaya., "A Torus Embedded Hypercube Scalable Interconnection Network for Parallel Architecture," IEEE explore conference publications, 2009.

[11] H. El-Rewini and M. Abd-El-Barr, " Advanced Computer Architecture and Parallel Processing," John Wiley \& Sons, Inc., Hoboken, New Jersey, 2005.

[12] R. Y. Wu, and C. H. Chen, "Node Disjoint Paths in Hierarchical Hypercube Networks," $20^{\text {th }}$ International 
Parallel and Distributed Processing Symposium, pp. 2529 Apr. 2006.

[13] W. Dally and B. Towles, "Principles and Practices of Interconnection Networks," Morgan Kaufmann Press, San Francisco, 2004.

[14] M. L. Jones, "Optical Networking Standards," J. Lightwave Technol., Vol. 22, No. 1, pp. 275-280, Jan., 2004.

[15] Ahmed Louri, “Optical Interconnection Networks for Scalable High-performance Parallel Computing Systems," Applied Optics, Vol. 38, No. 29, pp. 6176 - 6183, Oct. 1999.

\section{Author's Profile}

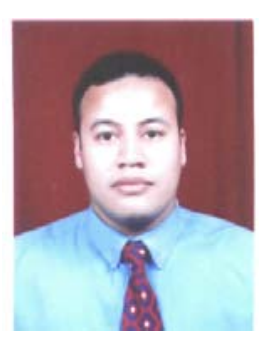

Dr. Ahmed Nabih Zaki Rashed was born in Menouf city, Menoufia State, Egypt country in 23 July, 1976. He received the B.Sc., M.Sc., and Ph.D. scientific degrees in the Electronics and Electrical Communications Engineering Department from Faculty of Electronic Engineering, Menoufia University in 1999, 2005, and 2010 respectively. Currently, his academic job carrier is a academic scientific lecturer in Electronics and Electrical Communications Engineering Department, Faculty of Electronic Engineering, Menoufia university, Menouf. Postal Menouf city code: 32951 , EGYPT.

His academic scientific research master science thesis has focused on polymer fibers in optical access communication systems. Moreover his academic scientific Ph. D. thesis has focused on recent applications in linear or nonlinear passive or active in optical networks. His interesting academic scientific research mainly focuses on transmission capacity of optical communication systems, high data rate and information product at long transmission distances of passive and active optical communication networks, optical wireless communications, radio over fiber communication systems, and optical network security and management. He has published many high scientific research papers in high quality and technical international journals in the field of advanced communication systems, optoelectronic devices, and passive optical access communication networks. He is Editorial board member in high academic scientific International research Journals. His areas of interest and experience in optical access communication systems, advanced optical communication networks, wireless optical access networks, analog communication systems, optical filters and Sensors, digital communication systems, optoelectronics devices, and advanced material science, network management systems, multimedia data base, communication network security, encryption and optical access computing systems. As well as he is a reviewer member and editorial board member in high Impacted academic scientific research international journals in the field of Electronics, Electrical communication, nonlinear optics, quantum optics, optoelectronics, advanced optical communication systems and networks. His personal electronic mail: ahmed_733@yahoo.com. 